See discussions, stats, and author profiles for this publication at: https://www.researchgate.net/publication/336346775

\title{
Strain localization analysis of Hill's orthotropic elastoplasticity Analytical results and numerical verification
}

Article in Computational Mechanics · October 2019

DOI: 10.1007/500466-019-01782-4

\section{CITATIONS}

4 authors, including:

\section{Miguel Cervera}

Universitat Politècnica de Catalunya

379 PUBLICATIONS 5,348 CITATIONS

SEE PROFILE

Michele Chiumenti

Universitat Politècnica de Catalunya

253 PUBLICATIONS 2,559 CITATIONS

SEE PROFILE

Some of the authors of this publication are also working on these related projects:

Project RIS3CAT LLAVOR3D TRANSPORT View project

Project Enhanced accuracy computational framework for strain localization and failure mechanisms (EACY) View project
Jian-Ying Wu

South China University of Technology

54 PUBLICATIONS 864 CITATIONS

SEE PROFILE 


\title{
Strain localization analysis of Hill's orthotropic elastoplasticity
}

\section{Analytical results and numerical verification}

\author{
Miguel Cervera ${ }^{1}$, Jian-Ying $\mathbf{W u}^{* 2}$, Michele Chiumenti ${ }^{1}$, Sungchul Kim ${ }^{1}$ \\ ${ }^{1}$ CIMnE, Technical University of Catalonia, Edificio C1, Campus Norte, Jordi Girona 1-3, 08034 Barcelona, Spain. \\ 2 State Key Laboratory of Subtropic Building Science, South China Univeristy of Technology, 510641 Guangzhou, China.
}

Received: date / Revised version: date

\begin{abstract}
In this work the strain localization analysis of Hill's orthotropic plasticity is addressed. In particular, the localization condition derived from the boundedness of stress rates together with Maxwell's kinematics is employed. Similarly to isotropic plasticity considered in our previous work, the plastic flow components on the discontinuity surface vanish upon strain localization. The resulting localization angles in orthotropic plastic materials are independent from the elastic constants, but rather, depend on the material parameters involved in the plastic flow in the material axes. Application of the above localization condition to Hill's orthotropic plasticity in 2-D plane stress and plane strain conditions yields closed-form solutions of the localization angles. It is found that the two discontinuity lines in plane strain conditions are always perpendicular to each other, and for the states of no shear stresses, the localization angle depends only on the tilt angle of the material axes with respect to the global ones. The analytical results are then validated by independent numerical simulations. The $B$-bar finite element is employed to deal with the incompressibility due to the purely isochoric plastic flow. For a strip under vertical stretching in plane stress and plane strain as well as Prandtl's problem of indentation by a flat rigid die in plane strain, numerical results are presented for both isotropic and orthotropic plasticity models with or without tilt angle. The influence of various parameters is studied. In all cases, the critical angles
\end{abstract}


predicted from the localization condition coincide with the numerical results, giving compelling supports to the analytical prognoses.

\section{Introduction}

Strain localization in solids is characterized by highly localized deformations and manifested by diverse phenomena of distinct length scales, e.g., dislocations of orders of microns in metals, cracks of order of millimeters in concrete, and shear bands of order ranging from millimeters to kilometers in granular and geological problems, etc. Being strain localization a prognosis of structural failure, it is of utmost significance to determine when strain localization occurs and quantify its adverse effects on the global response of structures.

Regarding rigid-plastic problems and shear driven, pressure independent flows, seminal works of Prandtl [1], Hencky [2,3] and Mandel [4] assumed the existence of "slip lines" and determined their directions by the "zero rate of extension" criterion. Later, Hill [5] revisited the similar problem and interpreted the family of "slip lines" as the characteristic curves (along which small disturbances propagate) of the hyperbolic plastic governing equations. With this method, the field of slip lines for typical metallurgical problems, e.g., sheet drawing and extrusion, piercing, strip-rolling, etc., were determined [6]. Note that in these early studies, elastic deformations were explicitly ignored, and perfectly incompressible behavior prior to shear driven plastic yielding was assumed.

For general elasto-plastic materials, as strain localization inevitably induces strain (weak) or even displacement (strong) discontinuities, the discontinuous bifurcation condition set forth by $[7,8,9,10]$ is customarily employed. More specifically, the necessary condition for strain localization in elastoplastic materials is identified upon the assumption of linear comparison solids (i.e., inelastic loading both inside and outside the localization band) and traction continuity $[11,12,13]$. Closed-form results for the localization angles have been obtained for 2-D plane stress and plane strain conditions in this way [13]. One noteworthy property of such results is that the localization angle depends on the elastic constants, e.g., Poisson's ratio. 
The classical discontinuous bifurcation analysis has been applied not only to weak discontinuities, but also to strong ones. For instance, $[14,15]$ used it to determine the discontinuity orientation, such that strong discontinuities can be embedded in standard finite elements. However, it was soon found that this discontinuous bifurcation condition by itself does not necessarily guarantee the occurrence of strong discontinuities, unless the strong discontinuity is properly regularized, involving also stress boundedness $[14,15,16,17]$. In particular, the fact that material points inside the discontinuity band undergo inelastic loading while those outside it unload elastically, is inconsistent with the assumption of linear comparison solids in which inelastic loading is assumed in both the bulk and localization band. Furthermore, due to the singular strain field caused by displacement discontinuities, the traction continuity condition alone is sufficient to guarantee neither stress locking-free results or the decohesion limit due to the mis-prediction of the discontinuity orientation $[18,19]$.

In order to overcome the above crucial but generally overlooked issue, the authors [18] proposed further exploiting the kinematic compatibility condition resulting from stress boundedness to determine the discontinuity orientation of von Mises $\left(J_{2}\right)$ plastic materials. It turns out that the condition for stress boundedness is more constrictive for the orientation of discontinuities than the localization condition based on singularity of the acoustic tensor. More specifically, a given discontinuity orientation $\boldsymbol{n}$ that satisfies the localization condition $\operatorname{det} \boldsymbol{Q}_{\mathrm{ep}}(H, \boldsymbol{n})=0$ for a given maximum softening parameter $H<0$, guarantee neither stress boundedness nor full decohesion in the final stage of the deformation process. Reversely, stress boundedness implies both satisfaction of the classical discontinuous bifurcation condition and also perfect decohesion in the softening or perfect plasticity cases.

Remarkably, for elasto-plastic materials the above kinematic compatibility based localization condition predicts localization angles which depend exclusively on the components of the flow strain tensor. This incorporates as a particular case Hills zero rate of extension" for the classical slip-line theory for rigid-plastic problems. Compared to those given by the discontinuous bifurcation condition, the localization angles are independent of the elastic constants as well as of the softening parameter, as the tangent elasto plastic tensor is not involved in the analysis. 
More recently, the authors $[20,21,22]$ have successfully extended the above strain localization analysis to a unified stress-based plastic-damage model with general (e.g., Rankine, von Mises, Mohr-Coulomb, DruckerPrager, and more complex elliptic, parabolic, hyperbolic, etc.) failure criteria and to strain-based elastic-damage models. The closed-form results were remarkably confirmed by independent numerical simulations [23] in which the analytical results are not used in finite element analyses. Moreover, not only the discontinuity orientation but also the corresponding localized cracking model, i.e., constitutive relations, evolution equations, tractionbased failure criterion, softening functions, etc., can be determined consistently from a given material model. With these work, the gap between continuous and discontinuous approaches for the modeling of localized failure in solids [20] has been largely bridged.

In many industrial applications, e.g., additive manufacturing, automotive rolling, etc., engineering materials like steel sheets, aluminum, wood, paper and stratified rocks, composites with oriented fibers, etc., exhibit strongly orthotropic behavior. Ever since the pioneering work of Hill [7], plasticity models with orthotropic yield functions, e.g., Hoffman model [24] and Tsai-Wu model [25], among many others, have been extensively studied; see [26]. However, most of the aforementioned work considered only strain localization in isotropic materials and very rare references deal with orthotropic ones. To the authors' best knowledge, it is only in [27] that strain localization in orthotropic plasticity models is considered to determine the upper bound load capacity of such materials. Nevertheless, the closed-form results for the localization angle are not available. Consequently, the failure modes have to be calibrated from finite element simulations. This deficiency is sometimes not acceptable since the numerical results can be sensitive to the mesh alignment, leading to ill-predictions of failure modes and global responses $[28,29,30,31]$.

For these reasons, this work addresses strain localization analysis in orthotropic elastoplastic materials. The objectives are four-fold: $(i)$ to show that our previously established localization condition also applies to materials with orthotropic elastic and plastic behavior; $(i i)$ to analyze strain localization of orthotropic plastic models and, in particular, to determine the localization angles in 2-D plane stress and plane strain conditions; (iii) to numerically verify the analytical results through independent finite element simulations, and (iv) to 
investigate the influence of various material parameters on strain localization in orthotropic materials. Though other orthotropic yield functions can be considered, Hill's quadratic one is adopted in this work as the prototype example due to its wide application.

The remainder of this paper is structured as follows. Section 2 addresses the localization condition of elastoplastic solids based on the boundedness of stress rates together with Maxwell's kinematics. Section 3 is devoted to the application of the derived localization condition to Hill's orthotropic plasticity. In particular, closed-form results of the localization angles under 2-D conditions of plane stress and plane strain are given. Numerical verification of the analytical results is presented in Section 4 using the $B$-bar finite elements to deal with the isochoric nature of the plastic flow. A horizontal slit under vertical stretch and the Prandtl punch test, with or without tilt angle between the material local axes and the global ones, are considered. The influence of various parameters on the localization angles is also studied. The most relevant conclusions are drawn in Section 5 to close the paper.

Notation. Compact tensor notation is used in the theoretical part of this paper. As general rules, scalars are denoted by italic light-face Greek or Latin letters (e.g. $a$ or $\lambda$ ); vectors, second- and fourth-order tensors are signified by italic boldface minuscule, majuscule and blackboard-bold majuscule characters like $\boldsymbol{a}, \boldsymbol{A}$ and $\mathbb{A}$, respectively. The inner products with single and double contractions are denoted by '.' and ' $:$ ', respectively, while the dyadic operator is signified by ' $\otimes$ '. The Voigt notation of vectors and second-order tensors is denoted by boldface minuscule and majuscule letters like $\mathbf{a}$ and $\mathbf{A}$, respectively.

\section{Strain localization of elastoplastic solids}

In this section, our previous work on strain localization in inelastic solids is briefly recalled and then particularized to elastoplastic materials. The resulting solution extends Hill's results for strictly incompressible rigid-plastic materials to general associated elasto-plastic materials, incompressible or not. Compared to the classical discontinuous bifurcation analysis $[7,8,9,11,12,13]$, not only traction continuity but also stress boundedness are guaranteed $[18,20,21,22]$ by reproducing Maxwell's discontinuity kinematics. 
Let us consider the domain $\Omega \subset \mathbb{R}^{n_{\mathrm{dim}}}\left(n_{\mathrm{dim}}=1,2,3\right)$ occupied by an elastoplastic solid with reference position vector $\boldsymbol{x} \in \mathbb{R}^{n_{\mathrm{dim}}}$. The boundary is denoted by $\Gamma \subset \mathbb{R}^{n_{\mathrm{dim}}-1}$, with an external unit normal vector $\boldsymbol{n}^{*}$. Deformations of the solid are characterized by the displacement field $\boldsymbol{u}: \Omega \rightarrow \mathbb{R}^{n_{\mathrm{dim}}}$ and the infinitesimal strain field $\boldsymbol{\epsilon}:=\nabla^{\mathrm{sym}} \boldsymbol{u}$, with $\nabla(\cdot)$ being the spatial gradient operator.

\subsection{Elastoplasticity model}

For an elastoplastic model, the constitutive relation is expressed in total form as

$$
\boldsymbol{\epsilon}=\epsilon^{\mathrm{e}}+\epsilon^{\mathrm{p}}, \quad \boldsymbol{\sigma}=\mathbb{E}^{0}: \epsilon^{\mathrm{e}}=\mathbb{E}^{0}:\left(\boldsymbol{\epsilon}-\epsilon^{\mathrm{p}}\right)
$$

where the second-order tensors $\boldsymbol{\epsilon}^{\mathrm{e}}$ and $\boldsymbol{\epsilon}^{\mathrm{p}}$ represent the elastic and plastic parts of the strain $\boldsymbol{\epsilon}$, respectively; the second-order stress tensor $\boldsymbol{\sigma}$ is related to the elastic strain $\boldsymbol{\epsilon}^{\mathrm{e}}$ by fourth-order elasticity tensor $\mathbb{E}^{0}$. Note that $\mathbb{E}^{0}$ may be isotropic or orthotropic in this work.

For the classical associated evolution law, the plastic strain rate is given by

$$
\dot{\boldsymbol{\epsilon}}^{\mathrm{p}}=\dot{\lambda} \boldsymbol{\Lambda}, \quad \dot{\kappa}=\dot{\lambda} h
$$

where $\dot{\lambda} \geq 0$ denotes the plastic multiplier, with $(\dot{)})$ being the time derivative; the derivatives $\boldsymbol{\Lambda}:=\partial \phi / \partial \boldsymbol{\sigma}$ and $h:=\partial \phi / \partial q$ are normal to the yield surface $\phi(\sigma, q)=0$, with $q$ being a stress-like internal variable (yield stress). In this work, Hill's orthotropic yield function is considered later in Eq. (3.1).

The corresponding constitutive relation in rate form then reads

$$
\dot{\boldsymbol{\sigma}}=\mathbb{E}^{0}:\left(\dot{\boldsymbol{\epsilon}}-\dot{\boldsymbol{\epsilon}}^{\mathrm{p}}\right)=\mathbb{E}^{\mathrm{ep}}: \dot{\boldsymbol{\epsilon}}
$$

where the fourth-order elastoplasticity tensor $\mathbb{E}^{\text {ep }}$ is expressed as

$$
\mathbb{E}^{\mathrm{ep}}=\mathbb{E}^{0}-\frac{\mathbb{E}^{0}: \boldsymbol{\Lambda} \otimes \boldsymbol{\Lambda}: \mathbb{E}^{0}}{\boldsymbol{\Lambda}: \mathbb{E}^{0}: \boldsymbol{\Lambda}+h \cdot H \cdot h}
$$

for the hardening/softening modulus $H:=\partial q / \partial \kappa$. 


\subsection{Discontinuity kinematics}

At the early stage of the deformation process, the standard continuum kinematics applies, in which both the velocity and strain rate fields are continuous and regular (bounded). For a perfectly or softened plastic solid, upon satisfaction of the yield condition $\phi(\sigma, q)=0$, the deformation can grow unbounded. In particular, two orthogonal families of curves (surfaces in 3D) form in 2-D conditions. These so-called slip lines or surfaces represent macroscopic phenomena occurring at the micro- or meso-level associated with strain localization and induce jumps in the strain rate or even velocity fields.

Velocity jumps can be described by strong discontinuities. As depicted in Figure 1(a), the interface $\mathcal{S}$ splits the solid $\Omega$ into two parts $\Omega^{+}$and $\Omega^{-}$, located "ahead of" and "behind" $\mathcal{S}$, respectively. The discontinuity orientation is characterized by a unit normal vector $\boldsymbol{n}$, pointing from $\Omega^{-}$to $\Omega^{+}$and fixed along time, i.e., $\boldsymbol{n}=\boldsymbol{O}$, with ( ) being the time derivative. Alternatively, strain discontinuities can be characterized by a discontinuity band $\mathcal{B}$ of finite width $b$, delimited by two surfaces $\mathcal{S}^{+}$and $\mathcal{S}^{-}$parallel to the discontinuity $\mathcal{S}$ as shown in Figure 1(b). As the bandwidth $b$ is a regularization parameter that can be made as small as desired, the strong discontinuity can be regarded as the limit of a regularized one, with a vanishing bandwidth $b \rightarrow 0$. Reciprocally, a discontinuity band can be regarded as the convenient regularization of a strong discontinuity.

Upon the above setting, the strain rate fields $\dot{\boldsymbol{\epsilon}}_{\text {int }}$ and $\dot{\boldsymbol{\epsilon}}_{\text {ext }}$ inside and outside the discontinuity band $\mathcal{B}$ verify the following Maxwell's compatibility condition [9]

$$
\dot{\boldsymbol{\epsilon}}_{\mathrm{int}}=\dot{\boldsymbol{\epsilon}}_{\mathrm{ext}}+(\dot{\boldsymbol{e}} \otimes \boldsymbol{n})^{\mathrm{sym}}
$$

or, equivalently,

$$
\llbracket \dot{\boldsymbol{\epsilon}} \rrbracket:=\dot{\boldsymbol{\epsilon}}_{\mathrm{int}}-\dot{\boldsymbol{\epsilon}}_{\mathrm{ext}}=(\dot{\boldsymbol{e}} \otimes \boldsymbol{n})^{\mathrm{sym}}
$$

where the inelastic deformation rate vector $\dot{\boldsymbol{e}}:=\dot{\boldsymbol{w}} / b$ is defined as the apparent velocity jump $\dot{\boldsymbol{w}}$ across the discontinuity band $\mathcal{B}$ normalized by the regularization length $b$; see Figure 2(a) for the strong discontinuity and Figure 2(b) for a regularized one, respectively. Note that the jump of strain rate, $\llbracket \dot{\boldsymbol{\epsilon}} \rrbracket$, is inversely proportional to $b$ for a regularized discontinuity or unbounded for a strong one. 


\subsection{Strain localization condition}

Upon strain localization, material points inside the discontinuity (band) undergo inelastic loading while those outside it unload elastically [16,18]. Accordingly, it follows from the constitutive relation $(2.3)$ that

$$
\dot{\boldsymbol{\sigma}}_{\mathrm{int}}=\mathbb{E}^{0}:\left(\dot{\boldsymbol{\epsilon}}_{\mathrm{int}}-\dot{\boldsymbol{\epsilon}}^{\mathrm{p}}\right), \quad \dot{\boldsymbol{\sigma}}_{\mathrm{ext}}=\mathbb{E}^{0}: \dot{\boldsymbol{\epsilon}}_{\mathrm{ext}}
$$

The resulting jump in the stress rate, $\llbracket \dot{\boldsymbol{\sigma}} \rrbracket$, is given by

$$
\llbracket \dot{\boldsymbol{\sigma}} \rrbracket=\dot{\boldsymbol{\sigma}}_{\mathrm{int}}-\dot{\boldsymbol{\sigma}}_{\mathrm{ext}}=\mathbb{E}^{0}:\left[(\dot{\boldsymbol{e}} \otimes \boldsymbol{n})^{\mathrm{sym}}-\dot{\boldsymbol{\epsilon}}^{\mathrm{p}}\right]
$$

The stress tensors and their rates have to remain bounded during the failure process to guarantee their physical

significance. Being $\dot{\boldsymbol{\sigma}}_{\text {int }}$ and $\dot{\boldsymbol{\sigma}}_{\text {ext }}$ both bounded, so is the jump $\llbracket \dot{\boldsymbol{\sigma}} \rrbracket$. Accordingly, the difference $(\dot{\boldsymbol{e}} \otimes \boldsymbol{n})^{\text {sym }}-\dot{\boldsymbol{\epsilon}}^{\mathrm{p}}$ has to be bounded, too. As the bandwidth $b$ is a regularization parameter that can be made as small as desired, the stress boundedness condition requires $[18,21]$

$$
\llbracket \dot{\boldsymbol{\epsilon}} \rrbracket=\dot{\boldsymbol{\epsilon}}^{\mathrm{p}}=(\dot{\boldsymbol{e}} \otimes \boldsymbol{n})^{\mathrm{sym}}
$$

That is, upon strain localization stress boundedness requires that the strain rate jump, defined as the difference in the strain rate fields between the interior/exterior points of the discontinuity (band) and characterized by Maxwell's compatibility condition, has to be completely plastic.

Remark 2.1 The kinematic constraint (2.9) implies that even though the corresponding strains are not, the stresses inside and outside the discontinuity band are continuous, i.e., $\llbracket \dot{\boldsymbol{\sigma}} \rrbracket=\boldsymbol{O}$. In this case, the traction continuity $\llbracket \dot{\boldsymbol{t}} \rrbracket=\boldsymbol{n} \cdot \llbracket \dot{\boldsymbol{\sigma}} \rrbracket$ is also guaranteed.

Remark 2.2 This stress boundedness/continuity holds for softened plasticity [18,20,21] and also for perfect plastic flows (incremental full decohesion). Similarly, the same condition guarantees that full decohesion $(\dot{\boldsymbol{\sigma}}=\boldsymbol{O})$ can be fulfilled with large plastic straining inside the discontinuity; see [18] for details. 


\subsection{Strain localization of plastic solids}

The kinematic constraint (2.9) implies the existence of a plastic flow vector $\gamma$ satisfying

$$
\dot{e}=\dot{\lambda} \gamma \quad \Longrightarrow \quad \boldsymbol{\Lambda}=(\gamma \otimes \boldsymbol{n})^{\mathrm{sym}}
$$

Let $(n, m, p)$ be discontinuity local axes, with $\boldsymbol{n}, \boldsymbol{m}$ and $\boldsymbol{p}$ being the normal vector, the in-plane and out-of-plane tangential ones of the discontinuity $\mathcal{S}$, respectively. It then follows that $[17,20,21]$

$$
\boldsymbol{\gamma}=2 \boldsymbol{n} \cdot \boldsymbol{\Lambda}-\boldsymbol{n} \Lambda_{n n}=\gamma_{n} \boldsymbol{n}+\gamma_{m} \boldsymbol{m}+\gamma_{p} \boldsymbol{p}
$$

where the components $\left(\gamma_{n}, \gamma_{m}, \gamma_{p}\right)$ of the plastic flow vector $\gamma$ are determined as

$$
\gamma_{n}:=\boldsymbol{\gamma} \cdot \boldsymbol{n}=\Lambda_{n n}, \quad \gamma_{m}:=\boldsymbol{\gamma} \cdot \boldsymbol{m}=2 \Lambda_{n m}, \quad \gamma_{p}:=\boldsymbol{\gamma} \cdot \boldsymbol{p}=2 \Lambda_{n p}
$$

Substitution of the above plastic flow vector $\boldsymbol{\gamma}$ back into the relation $(2.10)_{2}$ yields $[20,21]$

$$
\Lambda_{m m}\left(\boldsymbol{\theta}^{\mathrm{cr}}\right)=0, \quad \Lambda_{p p}\left(\boldsymbol{\theta}^{\mathrm{cr}}\right)=0, \quad \Lambda_{m p}\left(\boldsymbol{\theta}^{\mathrm{cr}}\right)=0
$$

where $\boldsymbol{\theta}^{\text {cr }}$ denote the localization angles upon which the kinematic constraint (2.10) is satisfied. Note that the components $\Lambda_{m m}, \Lambda_{p p}$ and $\Lambda_{m p}$ depend on the specific yield function $\phi(\boldsymbol{\sigma}, q)$; see Section 3.1 for the application to Hill's criterion.

Remark 2.3 As can be seen from Eq. (2.13), upon strain localization the plastic flow tensor evolves into a particular structure in terms of a localized flow vector and the discontinuity orientation. Accordingly, the tensorial flow components in the directions orthogonal to the discontinuity orientation have to vanish so that the consistent loading/unloading deformation states upon strain localization are correctly represented and slip lines or surfaces eventually form. This result is an extension of Hill's criterion of "zero rate of extension" for incompressible rigid-plastic materials $[5,6]$ to general elasto-plastic ones. In the following, we apply this procedure to determine the localization angle for Hill's orthotropic plastic materials. 
Remark 2.4 In the case of plane strain, the strain localization condition $\Lambda_{33}=\Lambda_{p p}=0$ is not necessarily fulfilled at the onset of plastic yield $\phi(\boldsymbol{\sigma}, q)=0$ with the out-of-plane stress given by $\epsilon_{33}=0$. Accordingly, except for very particular cases, strain localization cannot occur at the onset of plastic yielding. Rather, some (continuous) inelastic deformations and substantial rotation of the principal strain directions have to occur, until the localization condition $\Lambda_{33}=\Lambda_{p p}=0$ is fulfilled such that strain localization is set in motion and a strong (regularized) discontinuity forms; see the numerical results presented in [18] for the case of von Mises $\left(J_{2}\right)$ model with isotropic linear elasticity.

Remark 2.5 The above localization conditions allow developing a traction-based plastic model for the discontinuity (band). Both the orientation and the traction-based failure criterion can be determined a posteriori from the given stress-based counterpart; see $[32,20,21,22]$ for the details. However, this extra procedure is not considered in this work.

\section{Application to Hill's orthotropic plastic materials}

In this section the above strain localization condition is applied to Hill's orthotropic perfectly plastic materials [33]. In particular, closed-form solutions are obtained for the localization angle in the 2-D plane stress and plane strain conditions. Similarly, Hill [5] considered only the plane stress condition for rigid-plastic materials.

\subsection{Hill's orthotropic plasticity}

Without loss of generality, let us consider Hill's orthotropic yield criterion of the following form [33]

$$
\phi(\boldsymbol{\sigma})=F\left(\sigma_{11}-\sigma_{22}\right)^{2}+G\left(\sigma_{11}-\sigma_{33}\right)^{2}+H\left(\sigma_{22}-\sigma_{33}\right)^{2}+2 L \sigma_{12}^{2}+2 M \sigma_{13}^{2}+2 N \sigma_{23}^{2}-1 \leq 0
$$


with the material parameters $F, G, H, L, M$ and $N$ given by

$$
\begin{array}{ll}
F=\frac{1}{2}\left[\left(\frac{1}{\sigma_{Y, 11}}\right)^{2}+\left(\frac{1}{\sigma_{Y, 22}}\right)^{2}-\left(\frac{1}{\sigma_{Y, 33}}\right)^{2}\right], & L=\frac{1}{2}\left(\frac{1}{\sigma_{Y, 12}}\right)^{2} \\
G=\frac{1}{2}\left[\left(\frac{1}{\sigma_{Y, 11}}\right)^{2}+\left(\frac{1}{\sigma_{Y, 33}}\right)^{2}-\left(\frac{1}{\sigma_{Y, 22}}\right)^{2}\right], & M=\frac{1}{2}\left(\frac{1}{\sigma_{Y, 13}}\right)^{2} \\
H=\frac{1}{2}\left[\left(\frac{1}{\sigma_{Y, 22}}\right)^{2}+\left(\frac{1}{\sigma_{Y, 33}}\right)^{2}-\left(\frac{1}{\sigma_{Y, 11}}\right)^{2}\right], & N=\frac{1}{2}\left(\frac{1}{\sigma_{Y, 23}}\right)^{2}
\end{array}
$$

where $\sigma_{11}, \sigma_{22}, \sigma_{33}, \sigma_{12}, \sigma_{13}$ and $\sigma_{23}$ denote the stress components in the material local axes $(1,2,3)$, with those entities embellished by subscripts " $Y$ " representing the corresponding yield strengths.

The components $\Lambda_{i j}$ of the flow tensor $\boldsymbol{\Lambda}:=\mathrm{d} \phi / \mathrm{d} \boldsymbol{\sigma}$ are then expressed as

$$
\begin{aligned}
& \Lambda_{11}=\frac{\mathrm{d} \phi}{\mathrm{d} \sigma_{11}}=2(F+G) \sigma_{11}-2 F \sigma_{22}-2 G \sigma_{33} \\
& \Lambda_{22}=\frac{\mathrm{d} \phi}{\mathrm{d} \sigma_{22}}=2(F+H) \sigma_{22}-2 F \sigma_{11}-2 H \sigma_{33} \\
& \Lambda_{33}=\frac{\mathrm{d} \phi}{\mathrm{d} \sigma_{33}}=2(G+H) \sigma_{33}-2 G \sigma_{11}-2 H \sigma_{22} \\
& \Lambda_{12}=\Lambda_{21}=\frac{1}{2} \frac{\mathrm{d} \phi}{\mathrm{d} \sigma_{12}}=2 L \sigma_{12} \\
& \Lambda_{13}=\Lambda_{31}=\frac{1}{2} \frac{\mathrm{d} \phi}{\mathrm{d} \sigma_{13}}=2 M \sigma_{13} \\
& \Lambda_{23}=\Lambda_{32}=\frac{1}{2} \frac{\mathrm{d} \phi}{\mathrm{d} \sigma_{23}}=2 N \sigma_{23}
\end{aligned}
$$

Note that the identity $\operatorname{tr} \boldsymbol{\Lambda}=\Lambda_{11}+\Lambda_{22}+\Lambda_{33}=0$ always holds. That is, Hill's yield function leads to an isochoric (purely deviatoric) plastic flow.

Remark 3.1 von Mises's isotropic yield criterion is recovered for

$$
F=G=H=\frac{1}{2}\left(\frac{1}{\sigma_{Y}}\right)^{2}, \quad L=M=N=\frac{3}{2}\left(\frac{1}{\sigma_{Y}}\right)^{2}
$$

for the yield strength $\sigma_{Y}$.

\subsection{Localization angles}

In this section strain localization of a 2-D Hill's plastic solid $\Omega \subset \mathbb{R}^{2}$ is considered. In such 2-D cases, the discontinuity orientation can be characterized by the inclination angle (counter-clockwise) $\theta \in[-\pi / 2, \pi / 2]$ 
between the normal vector $\boldsymbol{n}$ and the material axis 1 ; see Figure 3 . Accordingly, the flow components $\Lambda_{m m}, \Lambda_{p p}$ can be expressed in terms of $\Lambda_{11}, \Lambda_{22}, \Lambda_{33}, \Lambda_{12}$ in the material local axes as follows

$$
\Lambda_{m m}=\Lambda_{11} \sin ^{2} \theta+\Lambda_{22} \cos ^{2} \theta+2 \Lambda_{12} \cos \theta \sin \theta, \quad \Lambda_{p p}=\Lambda_{33}
$$

Our objective is to derive explicitly the discontinuity angle $\theta^{\text {cr }}$ upon strain localization.

For a given stress-based failure criterion $\phi \leq 0$, the critical angle $\theta^{\text {cr }}$ can be determined explicitly from the projection relation (2.10) or, more specifically, from the kinematic constraints (2.13) applied to the flow components (3.5), i.e.,

$$
\begin{aligned}
& \Lambda_{m m}\left(\theta^{\mathrm{cr}}\right)=\cos ^{2} \theta^{\mathrm{cr}}\left(\Lambda_{11} \tan ^{2} \theta^{\mathrm{cr}}+2 \Lambda_{12} \tan \theta^{\mathrm{cr}}+\Lambda_{22}\right)=0 \\
& \Lambda_{p p}\left(\theta^{\mathrm{cr}}\right)=\Lambda_{33}=0
\end{aligned}
$$

Note that in $2-\mathrm{D}$ cases the other constraint $\Lambda_{m p}\left(\theta^{\mathrm{cr}}\right)=0$ is automatically satisfied.

It then follows from Eq. (3.6a) that

$$
\tan \theta^{\mathrm{cr}}=-\frac{\Lambda_{12}}{\Lambda_{11}} \pm \sqrt{\left(\frac{\Lambda_{12}}{\Lambda_{11}}\right)^{2}-\frac{\Lambda_{22}}{\Lambda_{11}}}
$$

where the flow components $\Lambda_{11}$ and $\Lambda_{22}$ given in Eq. (3.3) are further constrained by the condition (3.6b).

As can be seen, the above discontinuity angle $\theta^{\text {cr }}$ depends on the ratios $\Lambda_{22} / \Lambda_{11}$ and $/$ or $\Lambda_{12} / \Lambda_{11}$, or equivalently, the stress state, upon strain localization. In particular, the distinction between plane stress and plane strain states has to be made regarding the condition (3.6b).

Remark 3.2 For the above stress-based failure criterion, the resulting localization angle $\theta^{\text {cr }}$ does not depend on the elastic constants like Young's modulus and Poisson's ratio. This is in strong contrast to the results given from classical discontinuous bifurcation analysis $[11,12,13]$. In orthotropic materials, the elastic properties may well be orthotropic. The localization angle $\theta^{\text {cr }}$ does not depend on this feature either.

Remark 3.3 For the case of $\Lambda_{12}=0$, it follows that

$$
\tan \theta^{\mathrm{cr}}= \pm \sqrt{-\frac{\Lambda_{22}}{\Lambda_{11}}}
$$


This is the result we previously obtained for isotropic plasticity models $[18,20,21]$. Note that for orthotropic materials, the analysis needs to be considered in the material local axes.

Remark 3.4 The above discontinuity angle $\theta^{\mathrm{cr}}$ is expressed in terms of the flow components (or stresses) in the material local axes. Though the localization analysis is independent of the frame of reference, it is sometimes more convenient to use those stresses in the global axes $(x, y, z)$. To this end, we can considered the following transformation relation

$$
\left\{\begin{array}{l}
\sigma_{11} \\
\sigma_{22} \\
\sigma_{33} \\
\sigma_{12}
\end{array}\right\}=\left[\begin{array}{cccc}
\cos ^{2} \alpha & \sin ^{2} \alpha & 0 & -2 \cos \alpha \sin \alpha \\
\sin ^{2} \alpha & \cos ^{2} \alpha & 0 & 2 \cos \alpha \sin \alpha \\
0 & 0 & 1 & 0 \\
\cos \alpha \sin \alpha-\cos \alpha \sin \alpha & 0 \cos ^{2} \alpha-\sin ^{2} \alpha
\end{array}\right]\left\{\begin{array}{l}
\sigma_{x x} \\
\sigma_{y y} \\
\sigma_{z z} \\
\sigma_{x y}
\end{array}\right\}
$$

for the counter-clockwise tilt angle $\alpha$ between the global axis $x$ and the material local axis 1 .

3.2.1 Plane stress Let us consider plane stress conditions with $\sigma_{33}=\sigma_{p p}=0$. In this case, the relevant flow components in Eq. (3.3) become

$$
\begin{aligned}
& \Lambda_{11}=2(F+G) \sigma_{11}-2 F \sigma_{22} \\
& \Lambda_{22}=2(F+H) \sigma_{22}-2 F \sigma_{11} \\
& \Lambda_{12}=2 L \sigma_{12}
\end{aligned}
$$

As the condition (3.6b) is automatically satisfied, strain localization occurs at the same instant as the initial failure surface $\phi=0$ is reached and the localization angle $\theta^{\text {cr }}$ is determined straightforwardly from Eq. (3.7) together with the relations (3.10). It is seen from the transformation (3.9) that the localization angle $\theta^{\text {cr }}$ depends on the stresses in the global axes and on the tilt.

3.2.2 Plane strain In this case, the condition (3.6b) yields

$$
\Lambda_{p p}=\Lambda_{33}=0 \quad \Longrightarrow \quad \sigma_{33}=\frac{G \sigma_{11}+H \sigma_{22}}{G+H}, \quad \Lambda_{11}+\Lambda_{22}=-\Lambda_{33}=0
$$


Accordingly, Eq. (3.7) becomes

$$
\tan \theta^{\mathrm{cr}}=-\frac{\Lambda_{12}}{\Lambda_{11}} \pm \sqrt{\left(\frac{\Lambda_{12}}{\Lambda_{11}}\right)^{2}+1}
$$

where the flow components are expressed as

$$
\Lambda_{11}=-\Lambda_{22}=2 \frac{F G+F H+G H}{G+H}\left(\sigma_{11}-\sigma_{22}\right), \quad \Lambda_{12}=2 L \sigma_{12}
$$

or, equivalently,

$$
\frac{\Lambda_{12}}{\Lambda_{11}}=\frac{(G+H) L}{F G+F H+G H} \cdot \frac{\sigma_{12}}{\sigma_{11}-\sigma_{22}}
$$

Therefore, the localization angle $\theta^{\text {cr }}$ depends on the ratio $\sigma_{12} /\left(\sigma_{11}-\sigma_{22}\right)$; see Remark 3.7.

Remark 3.5 It follows from Eq. (3.12) that

$$
\tan \theta_{1}^{\mathrm{cr}} \cdot \tan \theta_{2}^{\mathrm{cr}}=-1 \quad \Longrightarrow \quad\left|\theta_{1}^{\mathrm{cr}}-\theta_{2}^{\mathrm{cr}}\right|=90^{\circ}
$$

Accordingly, the discontinuity lines are perpendicular to each other.

Remark 3.6 For the case of $\Lambda_{12}=0$, Eq. (3.12) becomes

$$
\tan \theta^{\mathrm{cr}}=1 \quad \Longrightarrow \quad \theta^{\mathrm{cr}}= \pm 45^{\circ}
$$

This is exactly the result obtained for isotropic plasticity $[18,20,21]$.

Remark 3.7 It follows from the transformation (3.9) that

$$
\frac{\sigma_{12}}{\sigma_{11}-\sigma_{22}}=\frac{1}{2} \frac{\left(\sigma_{x x}-\sigma_{y y}\right) \sin (2 \alpha)+2 \sigma_{x y} \cos (2 \alpha)}{\left(\sigma_{x x}-\sigma_{y y}\right) \cos (2 \alpha)-2 \sigma_{x y} \sin (2 \alpha)}
$$

Regarding the stresses in the global axes, the following two cases are of interest

$$
\frac{\sigma_{12}}{\sigma_{11}-\sigma_{22}}= \begin{cases}\frac{1}{2} \tan (2 \alpha) & \sigma_{x y}=0 \\ \frac{1}{2 \tan (2 \alpha)} & \sigma_{x x}=\sigma_{y y}\end{cases}
$$

In the first case, the principal directions are aligned with the global axes, while in the second one, they are at $45^{\circ}$ with respect to the global reference. In both cases, the localization angle $\theta^{\text {cr }}$ depends only on the tilt angle $\alpha$ of the material axes. 


\subsection{Particular examples}

In order to make the above results more clear, let us consider the stress state of vertical stretching, i.e.,

$$
\sigma_{x x}=0, \quad \sigma_{y y}=\sigma, \quad \sigma_{x y}=0
$$

along axis $y$.

For the plane stress condition, it follows from Eqs. (3.9) and (3.10) that

$$
\begin{aligned}
& \frac{\Lambda_{22}}{\Lambda_{11}}=\frac{(F+H) \cos ^{2} \alpha-F \sin ^{2} \alpha}{(F+G) \sin ^{2} \alpha-F \cos ^{2} \alpha} \\
& \frac{\Lambda_{12}}{\Lambda_{11}}=-\frac{L \cos \alpha \sin \alpha}{(F+G) \sin ^{2} \alpha-F \cos ^{2} \alpha}
\end{aligned}
$$

The localization angle $\theta^{\mathrm{cr}}$ is then determined from Eq. (3.7). In particular, for the case $\alpha=0$, it follows that

$$
\tan \theta^{\mathrm{cr}}= \pm \sqrt{-\frac{\Lambda_{22}}{\Lambda_{11}}}= \pm \sqrt{\frac{F+H}{F}}
$$

which depends only on the material plastic parameters $F$ and $H$. This result is coincident with that in [5] obtained from the "zero rate of extension".

For the plane strain condition, the localization angle $\theta^{\text {cr }}$ is determined from Eq. (3.12)

$$
\frac{\Lambda_{12}}{\Lambda_{11}}=\frac{1}{2} \frac{(G+H) L}{F G+F H+G H} \tan (2 \alpha)
$$

For the case $\alpha=0$, it follows that

$$
\tan \theta^{\mathrm{cr}}= \pm 1 \quad \Longrightarrow \quad \theta^{\mathrm{cr}}= \pm 45^{\circ}
$$

\section{Numerical verifications}

In this section the analytical results presented in Section 3 are numerically verified. It is stressed that the numerical verification is totally independent from the analytical results. That is, these results are not used in any way in finite element simulations. Perfect plasticity with null modulus $H=0$ is considered in this work, though the present strain localization analysis applies also to plastic-damage models with softening regimes. 
This is because the equations from which the localization angle is obtained, Eq. (2.13) and Eq. (3.7), depend only on the plastic flow components in the material local system; they do not depend on the softening modulus. Compelling results for isotropic elastoplastic models with softening regimes are shown in reference [21].

As seen in previous Sections, Hill's plastic flow is isochoric by definition, and for strain localization to take place the plastic flow needs to be well developed and, at that stage, the (incompressible) plastic component of the deformation is dominant over the elastic part. Standard displacement-based finite elements are not well suited to cope with this quasi-incompressibility situation and this blunder is more evident if low order finite elements are used. Mixed displacement/pressure $(\mathbf{u} / p)$ finite element formulations are far more suitable to tackle (quasi)-incompressible problems [34]. In previous works, the authors have used mixed displacement-pressure elements $[35,18]$ and strain-displacement ones [22] in the solution of strain localization problems in isochoric and quasi-isochoric situations.

In this work, orthotropic elasticity is addressed as well as orthotropic plasticity. In orthotropic elasticity, two interesting questions arise in contrast to isotropic elasticity. On the one hand, there is no simple scalar relation between the pressure (or mean stress) and the volumetric strain. This renders inapplicable most developments related to mixed $\mathbf{u} / p$ formulations. This is also the case of some related elements, like the widely used $Q 1 P 0$, where the discontinuous constant pressure is eliminated at element level to yield a final formulation in terms of displacements only. On the other hand, the term "incompressible material" results a contentious matter when referred to anisotropic solids, see [36] for a discussion on this subject. Fortunately, the B-bar method can be introduced to deal with anisotropic and non-linear media [37,38]. This method is adopted in this work.

\subsection{B-bar finite element}

In the standard displacement based finite element method, the strain field $\epsilon$ inside an element is related to the nodal displacements a by the strain-displacement matrix $\mathbf{B}$ (discrete symmetric gradient operator)

$$
\epsilon=\mathrm{Ba}
$$


where matrix $\mathbf{B}$ is expressed as

$$
\mathbf{B}=\left[\mathbf{B}_{1}, \ldots, \mathbf{B}_{i}, \ldots, \mathbf{B}_{n_{n}}\right]
$$

for $1 \leq i \leq n_{n}$, with $n_{n}$ being the number of nodes in the element. In general 3D cases, sub-matrix $\mathbf{B}_{i}$ and its volumetric part $\mathbf{B}_{i}^{\text {vol }}$ are expressed in Voigt's notation as

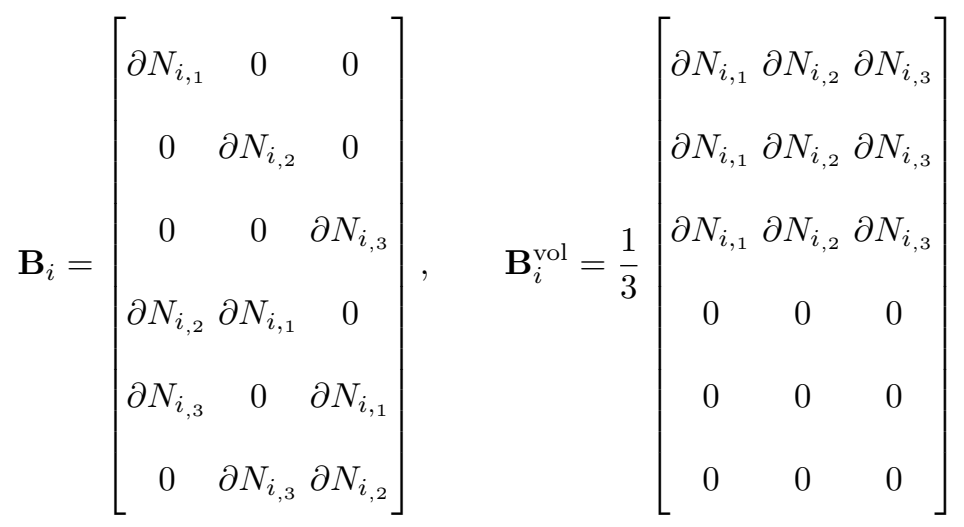

where $N_{i}$ is the shape function of node $i$ and $\partial N_{i, j}$ is its derivative with respect to the $j^{\text {th }}(1 \leq j \leq 3)$ Cartesian coordinate. The corresponding deviatoric part is then given by

$$
\mathbf{B}_{i}^{\text {dev }}=\mathbf{B}_{i}-\mathbf{B}_{i}^{\text {vol }}
$$

With these definitions, it follows that

$$
\boldsymbol{\epsilon}=\mathbf{B} \mathbf{a}=\left(\mathbf{B}^{\mathrm{dev}}+\mathbf{B}^{\mathrm{vol}}\right) \mathbf{a}=\boldsymbol{\epsilon}^{\mathrm{dev}}+\frac{1}{3} \epsilon^{\mathrm{vol}} \mathbf{1}
$$

where $\boldsymbol{\epsilon}^{\mathrm{dev}}=\operatorname{dev} \boldsymbol{\epsilon}$ and $\epsilon^{\mathrm{vol}}=\operatorname{tr} \boldsymbol{\epsilon}=\nabla \cdot \mathbf{u}$ are the deviatoric and volumetric strains, respectively.

Now, let $n_{g}$ be the number of integration points used to sample the strains and stresses and to integrate the internal nodal forces in the finite element. Then the average of the strain-displacement sub-matrices, $\overline{\mathbf{B}}_{i}^{\text {vol }}$, corresponding to node $i$, can be computed as

$$
\overline{\mathbf{B}}_{i}^{\mathrm{vol}}=\frac{1}{n_{g}} \sum_{k=1}^{n_{g}} \mathbf{B}_{i}^{\mathrm{vol}}
$$

The modified B-bar strain-displacement matrix is then defined as

$$
\overline{\mathbf{B}}_{i}=\mathbf{B}_{i}^{\mathrm{dev}}+\overline{\mathbf{B}}_{i}^{\mathrm{vol}}
$$


As explained in [37], the B-bar method is equivalent to the use of reduced integration for the volumetric part of the strain energy, while full integration is retained for the deviatoric contribution. Under given circumstances, a B-bar $Q 1$ element is identical to the mixed $Q 1 P 0$ element, but obtained through the deviatoric/volumetric split of the strains rather than the stresses.

\subsection{Example: Strip under vertical stretching}

In this section, a strip under vertical stretching is considered as shown in Figure 4. Following Hill [5] where characteristics are defined as "curves along which small disturbances propagates, a sharp horizontal slit is inserted in the strip to introduce the perturbation necessary to trigger strain localization. The far field stress state corresponds exactly to that given in Eq. (3.19). The analytical results presented in Section 3.3 are compared to the corresponding numerical ones.

4.2.1 Isotropic and orthotropic elasticity with von Mises plasticity. Let us consider the reference material of $J_{2}$ perfect plasticity, and isotropic elastic behavior with Young's modulus $E_{0}=1.0 \times 10^{7}$ MPa, Poisson's ratio $\nu_{0}=0.2$ and the yield strength $\sigma_{Y}=1.0 \times 10^{4} \mathrm{MPa}$.

Due to the facts $\sigma_{12}=0$ and $\Lambda_{12}=0$, the localization angle is given from Eq. (3.8), i.e.,

$$
\tan \theta^{\mathrm{cr}}=\left\{\begin{array}{lll} 
\pm \sqrt{2} & \text { Plane stress } \\
\pm 1 & \text { Plane strain }
\end{array} \quad \Longrightarrow \quad \theta^{\mathrm{cr}}= \begin{cases} \pm 54.74^{\circ} & \text { Plane stress } \\
\pm 45^{\circ} & \text { Plane strain }\end{cases}\right.
$$

The above results were obtained and numerically validated in previous works [18,21].

As shown in Figures 5 and 6 for the plane stress cases and Figures 7 and 8 for the plane strain cases, the localization angle $\theta^{\text {cr }}$ depends neither on Young's modulus nor on Poisson's ratio, even if they are varied in an orthotropic fashion.

4.2.2 Othotropic Hill material with no tilt. The Hill orthotropic plasticity material with the local axis 1 coincident with the global one $x$, i.e., $\alpha=0$, is considered. In this case, the analytical localization angle $\theta^{\mathrm{cr}}$ is given by Eqs. (3.21) and (3.23), respectively. 
Table 1 A strip under plane stress vertical stretching: Localization angles for various $\sigma_{Y, 11}$ while $\sigma_{Y, 22}=\sigma_{Y, 33}=\sigma_{Y}$ are fixed.

\begin{tabular}{|c|c|c|c|c|c|}
\hline Yield strength $\sigma_{Y, 11}$ & $F$ & $H$ & $\tan \theta^{\text {cr }}$ & $\theta^{\mathrm{cr}}$ & Angles of slip lines \\
\hline $0.75 \sigma_{Y}$ & $\frac{8}{9}\left(\frac{1}{\sigma_{Y}}\right)^{2}$ & $\frac{1}{9}\left(\frac{1}{\sigma_{Y}}\right)^{2}$ & $\pm \sqrt{\frac{9}{8}}$ & $\pm 46.69^{\circ}$ & $\pm 43.31^{\circ}$ \\
\hline$\sigma_{Y}$ (Isotropic) & $\frac{1}{2}\left(\frac{1}{\sigma_{Y}}\right)^{2}$ & $\frac{1}{2}\left(\frac{1}{\sigma_{Y}}\right)^{2}$ & $\pm \sqrt{2}$ & $\pm 54.74^{\circ}$ & $\pm 35.26^{\circ}$ \\
\hline $1.50 \sigma_{Y}$ & $\frac{2}{9}\left(\frac{1}{\sigma_{Y}}\right)^{2}$ & $\frac{7}{9}\left(\frac{1}{\sigma_{Y}}\right)^{2}$ & $\pm \sqrt{\frac{9}{2}}$ & $\pm 64.76^{\circ}$ & $\pm 25.24^{\circ}$ \\
\hline
\end{tabular}

Table 2 A strip under plane stress vertical stretching: Localization angles for various $\sigma_{Y, 22}$ while $\sigma_{Y, 11}=\sigma_{Y, 33}=\sigma_{Y}$ are fixed.

\begin{tabular}{|c|c|c|c|c|c|}
\hline Yield strength $\sigma_{Y, 22}$ & $F$ & $H$ & $\tan \theta^{\text {cr }}$ & $\theta^{\mathrm{cr}}$ & Angles of slip lines \\
\hline $0.75 \sigma_{Y}$ & $\frac{8}{9}\left(\frac{1}{\sigma_{Y}}\right)^{2}$ & $\frac{8}{9}\left(\frac{1}{\sigma_{Y}}\right)^{2}$ & $\pm \sqrt{2}$ & $\pm 54.74^{\circ}$ & $\pm 35.26^{\circ}$ \\
\hline$\sigma_{Y}$ (Isotropic) & $\frac{1}{2}\left(\frac{1}{\sigma_{Y}}\right)^{2}$ & $\frac{1}{2}\left(\frac{1}{\sigma_{Y}}\right)^{2}$ & $\pm \sqrt{2}$ & $\pm 54.74^{\circ}$ & $\pm 35.26^{\circ}$ \\
\hline $1.50 \sigma_{Y}$ & $\frac{2}{9}\left(\frac{1}{\sigma_{Y}}\right)^{2}$ & $\frac{2}{9}\left(\frac{1}{\sigma_{Y}}\right)^{2}$ & $\pm \sqrt{2}$ & $\pm 54.74^{\circ}$ & $\pm 35.26^{\circ}$ \\
\hline
\end{tabular}

- Plane stress: Let us first alter the yield strength $\sigma_{Y, 11}$ while the other two ones $\sigma_{Y, 22}$ and $\sigma_{Y, 33}$ are both fixed as $\sigma_{Y}$. The analytical results are summarized in Tables 1 and 2, against the numerical contours of the equivalent plastic strain shown in Figure 9.

As can be seen, the alteration of the yield strength $\sigma_{Y, 11}$ yields distinct localization angles for the slit under vertical stretching. Comparatively, alteration of $\sigma_{Y, 22}$ does not vary the localization angle since the slit is stretched along axis $y$.

- Plane strain: It follows from the analytical result Eq. (3.23) that the localization angle $\theta^{\text {cr }}$ is fixed as $45^{\circ}$ regardless the alteration of the material yield strengths. This conclusion is validated from the numerical contours presented in Figure 10. 
For all cases, the analytical results are coincident with the numerical ones as expected.

4.2.3 Othotropic Hill material with tilt. Let us now discuss the Hill orthotropic plasticity with the material local axis 1 different from the global one $x$, i.e., $\alpha \neq 0$. Similarly, the plane stress and plane strain conditions have to be discriminated.

- Plane stress: The analytical localization angle $\theta^{\mathrm{cr}}$ is given by Eq. (3.7) together with the ratios (3.20). In particular, some analytical results are summarized in Tables 3, 4, 5 and 6 . The numerical contours of the equivalent plastic strain are shown in Figures 11 to 14.

As can be seen, variation of the yield strengths in both directions yields distinct localization angles.

- Plane strain: Some of the analytical localization angles $\theta^{\text {cr }}$ given by Eq. (3.12) together with the ratios (3.22) are summarized in Tables 7 and 8. The corresponding numerical contours of the equivalent plastic strain are shown in Figures 15 and 16.

Compared to the results of plane stress, the variation of the yield strength in both directions has the same influences on the localization angles.

In all cases, the analytical results are reproduced by the numerical ones. Note again that the above results given from localization analyses are independent of the frame of reference. However, if the material axes are not aligned with the direction of straining, the strip is no longer of uniaxial straining in the material axes. Accordingly, considering material tilting with respect to the direction of straining verifies the results obtained for multi-directional straining.

\subsection{Example: Indentation by a flat rigid die}

The second example is the indentation by a flat rigid die shown in Figure 17. This is a well-known 2-D plane strain problem usually used in the literature to test the ability of plasticity model to capture the failure modes; see also [5]. 
Table 3 A strip under plane stress vertical stretching: Localization angles for $\sigma_{Y, 11}=1.50 \sigma_{Y}$ at various tilts $\alpha$.

\begin{tabular}{l|l|l|l|l}
\hline Tilt $\alpha$ & $\tan \theta^{\text {cr }}$ & $\theta^{\text {cr }}$ & $\theta^{\text {cr }}+\alpha$ & Angles of slip lines \\
\hline $0^{\circ}$ & $-2.1213 ; 2.1213$ & $64.8^{\circ} ;-64.8^{\circ}$ & $64.8^{\circ} ;-64.8^{\circ}$ & $-25.2^{\circ} ; 25.2^{\circ}$ \\
$30^{\circ}$ & $-23.906 ; 0.5229$ & $-87.6^{\circ} ; 27.6^{\circ}$ & $57.6^{\circ} ;-57.6^{\circ}$ & $-32.4^{\circ} ; 32.4^{\circ}$ \\
$45^{\circ}$ & $0.2644 ; 13.236$ & $14.8^{\circ} ; 85.7^{\circ}$ & $59.8^{\circ} ; 130.7^{\circ}$ & $-30.2^{\circ} ; 40.7^{\circ}$ \\
$60^{\circ}$ & $0.0651 ; 4.6115$ & $3.7^{\circ} ; 77.8^{\circ}$ & $63.7^{\circ} ; 137.8^{\circ}$ & $-26.3^{\circ} ; 47.8^{\circ}$ \\
\hline
\end{tabular}

Table 4 A strip under plane stress vertical stretching: Localization angles for $\sigma_{Y, 11}=0.75 \sigma_{Y}$ at various tilts $\alpha$.

\begin{tabular}{l|l|l|l|l}
\hline Tilt $\alpha$ & $\tan \theta^{\text {cr }}$ & $\theta^{\text {cr }}$ & $\theta^{\text {cr }}+\alpha$ & Angles of slip lines \\
\hline $0^{\circ}$ & $-1.0607 ; 1.0607$ & $46.7^{\circ} ;-46.7^{\circ}$ & $46.7^{\circ} ;-46.7^{\circ}$ & $-43.3^{\circ} ; 43.3^{\circ}$ \\
$30^{\circ}$ & $-6.2271 ; 0.3814$ & $-80.9^{\circ} ; 20.9^{\circ}$ & $50.9^{\circ} ;-50.9^{\circ}$ & $-39.1^{\circ} ; 39.1^{\circ}$ \\
$45^{\circ}$ & $0.0375 ; 3.3375$ & $2.1^{\circ} ; 73,3^{\circ}$ & $47.1^{\circ} ; 118.3^{\circ}$ & $-42.9^{\circ} ; 28.3^{\circ}$ \\
$60^{\circ}$ & $-0.2620 ; 1.4312$ & $-14.7^{\circ} ; 55.1^{\circ}$ & $45.3^{\circ} ; 115.1^{\circ}$ & $-44.7^{\circ} ; 25.1^{\circ}$ \\
\hline
\end{tabular}

Table 5 A strip under plane stress vertical stretching: Localization angles for $\sigma_{Y, 22}=1.50 \sigma_{Y}$ at various tilts $\alpha$.

\begin{tabular}{l|l|l|l|l}
\hline Tilt $\alpha$ & $\tan \theta^{\text {cr }}$ & $\theta^{\text {cr }}$ & $\theta^{\text {cr }}+\alpha$ & Angles of slip lines \\
\hline $0^{\circ}$ & $-1.1414 ; 1.1414$ & $54.7^{\circ} ;-54.7^{\circ}$ & $54.7^{\circ} ;-54.7^{\circ}$ & $-35.3^{\circ} ; 35.3^{\circ}$ \\
$30^{\circ}$ & $0.2169 ; 15.3716$ & $12.2^{\circ} ; 86.3^{\circ}$ & $42.2^{\circ} ; 116.3^{\circ}$ & $-47.8^{\circ} ; 26.3^{\circ}$ \\
$45^{\circ}$ & $0.0756 ; 3.7816$ & $4.3^{\circ} ; 75.2^{\circ}$ & $49.3^{\circ} ; 120.2^{\circ}$ & $-40.7^{\circ} ; 30.2^{\circ}$ \\
$60^{\circ}$ & $-0.0418 ; 1.9124$ & $-2.4^{\circ} ; 62.4^{\circ}$ & $57.6^{\circ} ; 122.4^{\circ}$ & $-32.4^{\circ} ; 32.4^{\circ}$ \\
\hline
\end{tabular}

Table 6 A strip under plane stress vertical stretching: Localization angles for $\sigma_{Y, 22}=0.75 \sigma_{Y}$ at various tilts $\alpha$.

\begin{tabular}{l|l|l|l|l}
\hline Tilt $\alpha$ & $\tan \theta^{\text {cr }}$ & $\theta^{\text {cr }}$ & $\theta^{\text {cr }}+\alpha$ & Angles of slip lines \\
\hline $0^{\circ}$ & $-1.1414 ; 1.1414$ & $54.7^{\circ} ;-54.7^{\circ}$ & $54.7^{\circ} ;-54.7^{\circ}$ & $-35.3^{\circ} ; 35.3^{\circ}$ \\
$30^{\circ}$ & $-3.8164 ; 0.6987$ & $-75.3^{\circ} ; 34.9^{\circ}$ & $-45.3^{\circ} ; 64.9^{\circ}$ & $44.7^{\circ} ;-25.1^{\circ}$ \\
$45^{\circ}$ & $0.2996 ; 26.700$ & $16.7^{\circ} ; 87.9^{\circ}$ & $61.7^{\circ} ; 132.9^{\circ}$ & $-28.3^{\circ} ; 42.9^{\circ}$ \\
$60^{\circ}$ & $-0.1606 ; 2.6219$ & $-9.1^{\circ} ; 69.1^{\circ}$ & $50.9^{\circ} ; 129.1^{\circ}$ & $39.1^{\circ} ; 39.1^{\circ}$ \\
\hline
\end{tabular}


Table 7 A strip under plane strain vertical stretching: Localization angles for $\sigma_{Y, 11}=1.50 \sigma_{Y}$ or $\sigma_{Y, 22}=1.50 \sigma_{Y}$ at various tilts $\alpha$.

\begin{tabular}{l|l|l|l|l}
\hline Tilt $\alpha$ & $\tan \theta^{\text {cr }}$ & $\theta^{\text {cr }}$ & $\theta^{\text {cr }}+\alpha$ & Angles of slip lines \\
\hline $0^{\circ}$ & $-1.0000 ; 1.0000$ & $45.0^{\circ} ;-45.0^{\circ}$ & $45.0^{\circ} ;-45.0^{\circ}$ & $-45.0^{\circ} ; 45.0^{\circ}$ \\
$30^{\circ}$ & $-6.7251 ; 0.1487$ & $-81.5^{\circ} ; 8.5^{\circ}$ & $-51.5^{\circ} ; 38.5^{\circ}$ & $38.5^{\circ} ;-51.5^{\circ}$ \\
$45^{\circ}$ & $0 ;-\infty$ & $0.0^{\circ} ;-90.0^{\circ}$ & $45.0^{\circ} ;-45.0^{\circ}$ & $-45.0^{\circ} ; 45.0^{\circ}$ \\
$60^{\circ}$ & $-0.1487 ; 6.7251$ & $-8.5^{\circ} ; 81.5^{\circ}$ & $51.5^{\circ} ; 141.5^{\circ}$ & $-38.5^{\circ} ; 51.5^{\circ}$ \\
\hline
\end{tabular}

Table 8 A strip under plane strain vertical stretching: Localization angles for $\sigma_{Y, 11}=0.75 \sigma_{Y}$ or $\sigma_{Y, 22}=0.75 \sigma_{Y}$ at various tilts $\alpha$.

\begin{tabular}{l|l|l|l|l}
\hline Tilt $\alpha$ & $\tan \theta^{\text {cr }}$ & $\theta^{\text {cr }}$ & $\theta^{\text {cr }}+\alpha$ & Angles of slip lines \\
\hline $0^{\circ}$ & $-1.0000 ; 1.0000$ & $45.0^{\circ} ;-45.0^{\circ}$ & $45.0^{\circ} ;-45.0^{\circ}$ & $-45.0^{\circ} ; 45.0^{\circ}$ \\
$30^{\circ}$ & $-2.9675 ; 0.3370$ & $-71.4^{\circ} ; 18.6^{\circ}$ & $-41.4^{\circ} ; 48.6^{\circ}$ & $48.6^{\circ} ;-41.4^{\circ}$ \\
$45^{\circ}$ & $0 ;-\infty$ & $0.0^{\circ} ;-90.0^{\circ}$ & $45.0^{\circ} ;-45.0^{\circ}$ & $-45.0^{\circ} ; 45.0^{\circ}$ \\
$60^{\circ}$ & $-0.3370 ; 2.9675$ & $-18.6^{\circ} ; 71.4^{\circ}$ & $41.4^{\circ} ; 131.4^{\circ}$ & $-48.6^{\circ} ; 41.4^{\circ}$ \\
\hline
\end{tabular}

As shown in Figure 18, the material right under the rigid die is almost under uniaxial vertical loading in the global axes, i.e., $\sigma_{x y}=0$. In accordance with Remark 3.7, the localization angle $\theta^{\text {cr }}$ is determined from Eq. (3.12) with the following ratio

$$
\frac{\Lambda_{12}}{\Lambda_{11}}=\frac{1}{2} \frac{(G+H) L}{F G+F H+G H} \tan (2 \alpha)
$$

for the tilt $\alpha$. 
4.3.1 Isotropic von Mises material. Similarly as before, let us first consider the reference isotropic material of $J_{2}$ perfect plasticity, with Young's modulus $E_{0}=1.0 \times 10^{7} \mathrm{MPa}$, Poisson's ratio $\nu_{0}=0.2$ and the yield strength $\sigma_{Y}=1.0 \times 10^{4} \mathrm{MPa}$

As shown in Figure 19, the localization angle is fixed $\theta^{\mathrm{cr}}=45^{\circ}$ regardless of the elastic constants. It is worthy noting that the failure modes are symmetric and corresponds to the claimed Prandtl's solution.

4.3.2 Orthotropic Hill material with no tilt. It follows from $\alpha=0$ that $\Lambda_{12} / \Lambda_{11}=0$. Accordingly, the localization angle is also fixed as $\theta^{\mathrm{cr}}=45^{\circ}$ regardless of the material yield strength; see Figure 20 for the numerical results.

4.3.3 Orthotropic Hill material with tilt. As the localization angle depends only on the tilt, regardless of the stresses, the results coincide with those for the slit under plane strain vertical stretching. Therefore, the analytical localization angles summarized in Tables 7 and 8 also apply here. The numerical results presented in Figures 21 and 22 validate this conclusion.

As can be seen, due to the tilt of the material axes the failure modes are no longer symmetric. Note that the results for $\alpha=45^{\circ}$ are identical to those for $\alpha=0^{\circ}$ and the results for $\alpha=30^{\circ}$ are symmetric to those for $\alpha=60^{\circ}$ in both figures.

Note that in all cases the obtained results agree with those of Hill [5] for rigid-plastic materials.

\section{Conclusions}

In this work the strain localization analysis of Hill's orthotropic plasticity is addressed. Similarly to our previous work on isotropic plastic or damage models, the localization condition is derived from the boundedness of stress rates together with Maxwell's kinematics. That is, the plastic flow components perpendicular to the discontinuity normal vector vanish upon strain localization. Compared to the classical work based on the discontinuous bifurcation analysis, in the material axes the localization angles are independent from the elastic constants, but rather, they depend exclusively on the material parameters involved in the plastic yield function. This turns out 
to be coincident with Hill's results for strictly incompressible rigid-plastic problems, extending them to general elasto-plastic materials.

In 2-D plane stress and plane strain situations, application of the above localization condition to Hill's orthotropic plasticity yields closed-form solutions of the localization angles. In particular, the discontinuity lines in plane strain conditions are always perpendicular to each other, and the localization angle depends only on the tite angle of the material axes for the case of shear stress free states.

The analytical results are validated independently by numerical simulations. Being the plastic flow purely isochoric, the $B$-bar finite element is employed to deal with the incompressibility of the plastic flow. Regarding a horizontal slit under vertical stretching and Prandtl's punch test in plane strain, numerical results are presented for both the isotropic plasticity and the orthotropic one with or without tilt angle between the material axes and the global ones. In all cases, the critical angles predicted from the localization condition coincide with those given by numerical simulations. Interestingly, as for Prandtl's punch test in plane strain the material right under the rigid footing is almost free of shear stresses, the localization angles are also independent from the stress state and can be determined as those for a slit under vertical stretching.

\section{Acknowledgments}

Financial support from the Spanish Government-MINECO-Proyectos de I + D (Excelencia)-DPI2017-85998-PADaMANT-Computational Framework for Additive Manufacturing of Titanium Alloy and the Catalan Government ACCIÓ - Ris3cat Transport and $\mathrm{PRO}^{2}$ Project is gratefully acknowledged. This work is also supported by the National Natural Science Foundation of China (51878294; 51678246), the State Key Laboratory of Subtropical Building Science (2018ZC04) and the Funding for Central Universities (2018PY20) to J.Y. Wu.

\section{References}

1. L. Prandtl. Über die häete plastistischer körper. Nachr. Ges. Wisensch, Göttingen, math. phys. Klasse, pages 74-85, 1920. 
2. H. Hencky. Über einige statisch bestimmte fälle des gleichgewichts in plastischen körpern. Z. Angew. Math. Mech., $3: 241-251,1923$.

3. H. Hencky. Zur theorie plastischer deformationen und der hierdurch im material hervorgerufenen nachspannungen. Z. Angew. Math. Mech., 4:323-334, 1924.

4. J. Mandel. Equilibre par trasches planes des solides à la limite d'écoulement. PhD thesis, Thèse, Paris, 1942.

5. R. Hill. The Mathematical Theory of Plasticity. Oxford University Press, New York, 1950.

6. R. Hill. On discontinuous plastic states, with special reference to localized necking in thin sheets. J. Mech. Phys. Solids, 1:19-30, 1952.

7. R. Hill. General theory of uniqueness and stability of elasto-plastic solids. J. Mech. Phys. Solids, 6:236-249, 1958.

8. R. Hill. Acceleration waves in solids. J. Mech. Phys. Solids, 10:1-16, 1962.

9. T.Y. Thomas. Plastic Flow and Fracture of Solids. Academic Press, New York, 1961.

10. J. R. Rice. A path independent integral and the approximate analysis of strain cncentrations by notches and cracks. J. Appl. Mech.-T. ASME, 35:379-386, 1968.

11. J. W. Rudnicki and J. R. Rice. Conditions of the localization of deformation in pressure-sensitive dilatant material. J. Mech. Phys. Solids, 23:371-394, 1975.

12. J. R. Rice and J. W. Rudnicki. A note on some features of the theory of localization of deformation. Int. J. Solids Structures, 16:597-605, 1980.

13. K. Runesson, N.S. Ottosen, and D. Peric. Discontinuous bifurcations of elastic-plastic solutions at plane stress and plane strain. Int. J. Plast., 7:99-121, 1991.

14. J.C. Simó, J. Oliver, and F. Armero. An analysis of strong discontinuities induced by strain-softening in rateindependent inelastic solids. Comput. Mech., 12:277-296, 1993.

15. J. Oliver. Modeling strong discontinuities in solid mechanics via strain softening constitutive equations. part i: Fundamentals; part ii: Numerical simulation. International Journal for Numerical Methods in Engineering, 39:35753600; 3601-3623, 1996.

16. J. Oliver, M. Cervera, and O. Manzoli. Strong discontinuities and continuum plasticity models: the strong discontinuity approach. Int. J. Plast., 15:319-351, 1999.

17. J. Oliver. On the discrete constitutive models induced by strong discontinuity kinematics and continuum constitutive equations. International Journal of Solids and Structures, 37:7207-7229, 2000. 
18. M. Cervera, M. Chiumenti, and D. Di Capua. Benchmarking on bifurcation and localization in $j_{2}$ plasticity for plane stress and plane strain conditions. Comput. Methods Appl. Mech. Eng., 241244:206224, 2012.

19. J. Oliver, A. E. Huespe, and I. F. Dias. Strain localization, strong discontinuities and material fracture: Matches and mismatches. Comput. Methods Appl. Mech. Eng., 241:323-336, 2006.

20. J. Y. Wu and M. Cervera. On the equivalence between traction- and stress-based approaches for the modeling of localized failure in solids. Journal of the Mechanics and Physics of Solids, 82:137-163, 2015.

21. J. Y. Wu and M. Cervera. A thermodynamically consistent plastic-damage framework for localized failure in quasibrittle solids: Material model and strain localization analysis. International Journal of Solids and Structures, 88$89: 227-247,2016$.

22. J. Y. Wu and M. Cervera. Strain localization of elastic-damaging frictional-cohesive materials: Analytical results and numerical verification. Materials, 10:434; doi:10.3390/ma10040434, 2017.

23. M. Cervera, M. Chiumenti, L. Benedetti, and R. Codina. Mixed stabilized finite element methods in nonlinear solid mechanics. part iii: Compressible and incompressible plasticity. Comput. Methods Appl. Mech. Eng., 285:752-775, 2015.

24. O. Hoffman. The brittle strength of orthotropic materials. J. Comp. Mater., 1:200-206, 1967.

25. S. W. Tsai and E. M. Wu. A general theory of strength for anisotropic materials. J. Comp. Mater., 5:58-80, 1971.

26. S. Oller, E. Car, and J. Lubliner. Definition of a general implicit orthotropic yield criterion. Comput. Methods Appl. Mech. Engrg., 192:895-912, 2003.

27. M. Li, J. Füssl, M. Lukacevic, and J. Eberhardsteiner. A numerical upper bound formulation with sensibly-arranged velocity discontinuities and orthotropic material strength behavior. Journal of Theoretical and Applied Mechanics, $56(2): 417-433,2018$.

28. J.G. Rots, P. Nauta, G.M.A. Kusters, and J. Blaauwendraad. Smeared crack approach and fracture localization in concrete. Heron, 30:1-47, 1985.

29. M. Jirásek and T. Zimmermann. Analysis of rotating crack model. J. Eng. Mech., ASCE, 124(8):842-851, 1998.

30. M. Cervera. A smeared-embedded mesh-corrected damage model for tensile cracking. Int. J. Numer. Meth. Engng., $76: 1930-1954,2008$.

31. M. Cervera. An orthotropic mesh corrected crack model. Comput. Methods Appl. Mech. Engrg., 197:1603-1619, 2008. 
32. J. Y. Wu and M. Cervera. Strain localization and failure mechanics for elastoplastic damage solids. Monograph CIMNE M147, Barrcelona, Spain, 2014.

33. R. Hill. A theory of the yielding and plastic flow of anisotropic metals. Proc. Roy. Soc. A, 193:281-297, 1948.

34. J.C. Simó and T.J.R. Hughes. Computational inelasticity. Springer, New York, 1998.

35. M. Cervera and M. Chiumenti. Size effect and localization in $j_{2}$ plasticity. International Journal of Solids and Structures, 46:3301-3312, 2009.

36. C. A. Felippa and E. Oñate. Stress, strain and energy splittings for anisotropic solids under volumetric constraints. Computers \& Structures, 81(13):1343-1357, 2003.

37. T. J. R. Hughes. Generalization of selective intertegration procedures to anisotropic and nonlinear media. International Journal for Numerical Methods in Engineering, 15(9):1413-1418, 1980.

38. T.R.J. Hughes. The finite element method. Linear Static and Dynamic Finite Element Analysis. Dover Publications Inc., Mineaola, New York, 2000. 


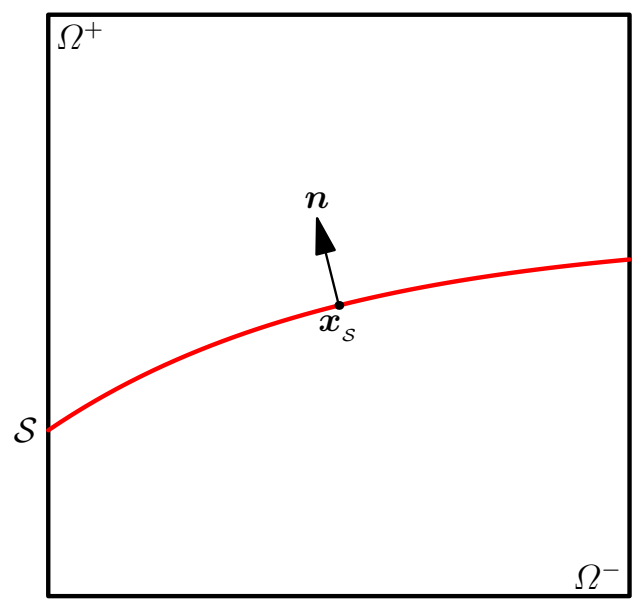

(a) Strong discontinuity

Fig. 1 Strong and regularized discontinuities in a solid

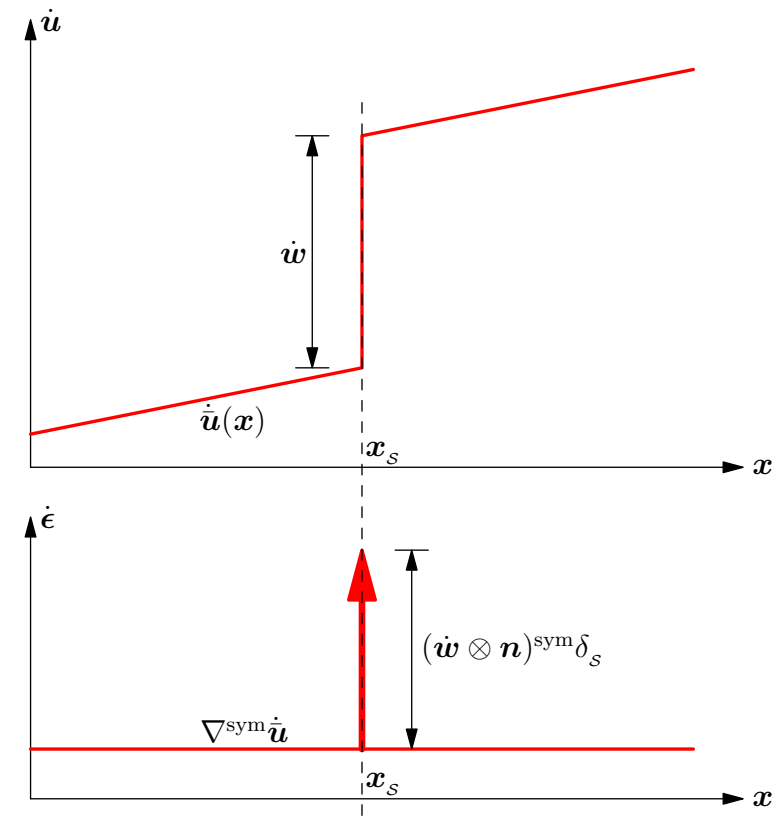

(a) Velocity/strain rate fields around a strong discontinuity

Fig. 2 Kinematics of strong and regularized discontinuities

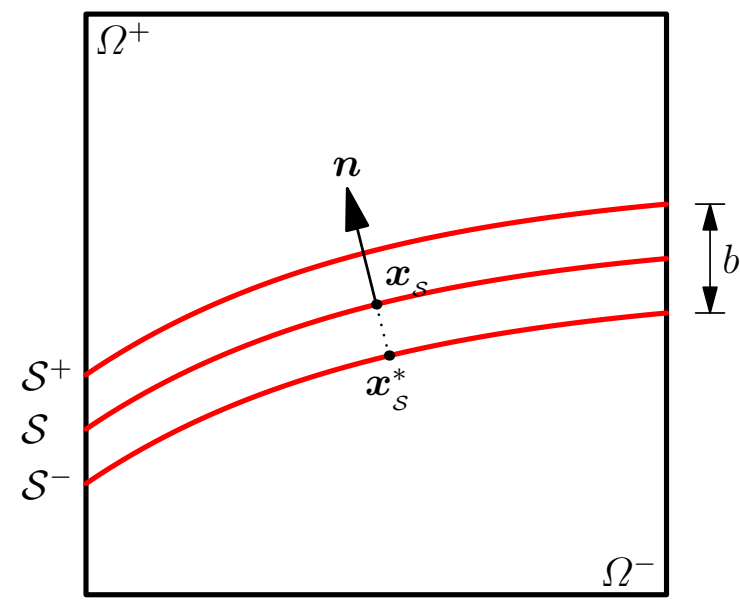

(b) Regularized discontinuity

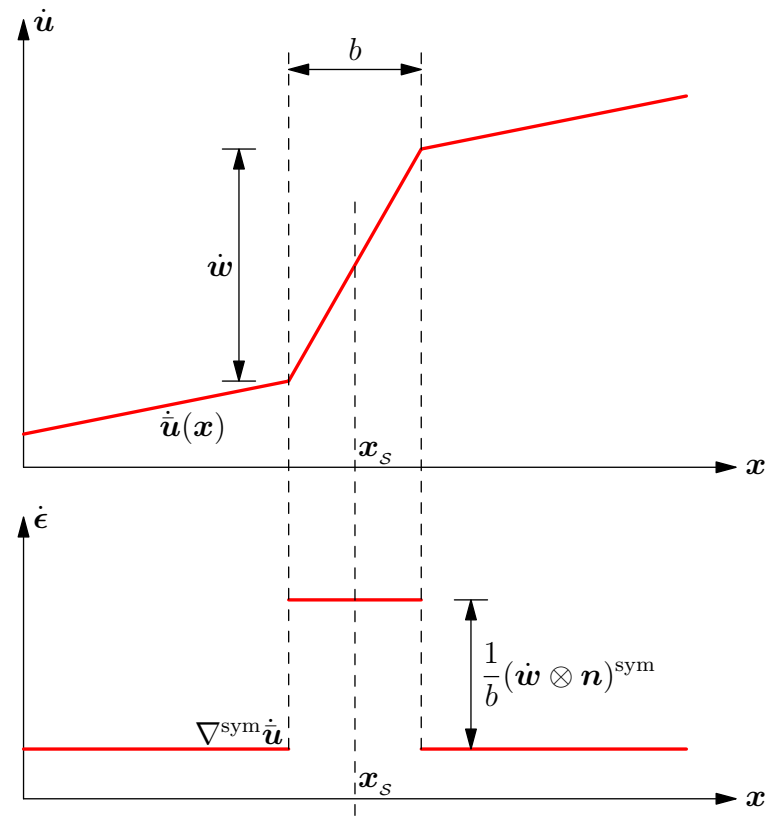

(b) Velocity/strain rate fields around a regularized discontinuity 


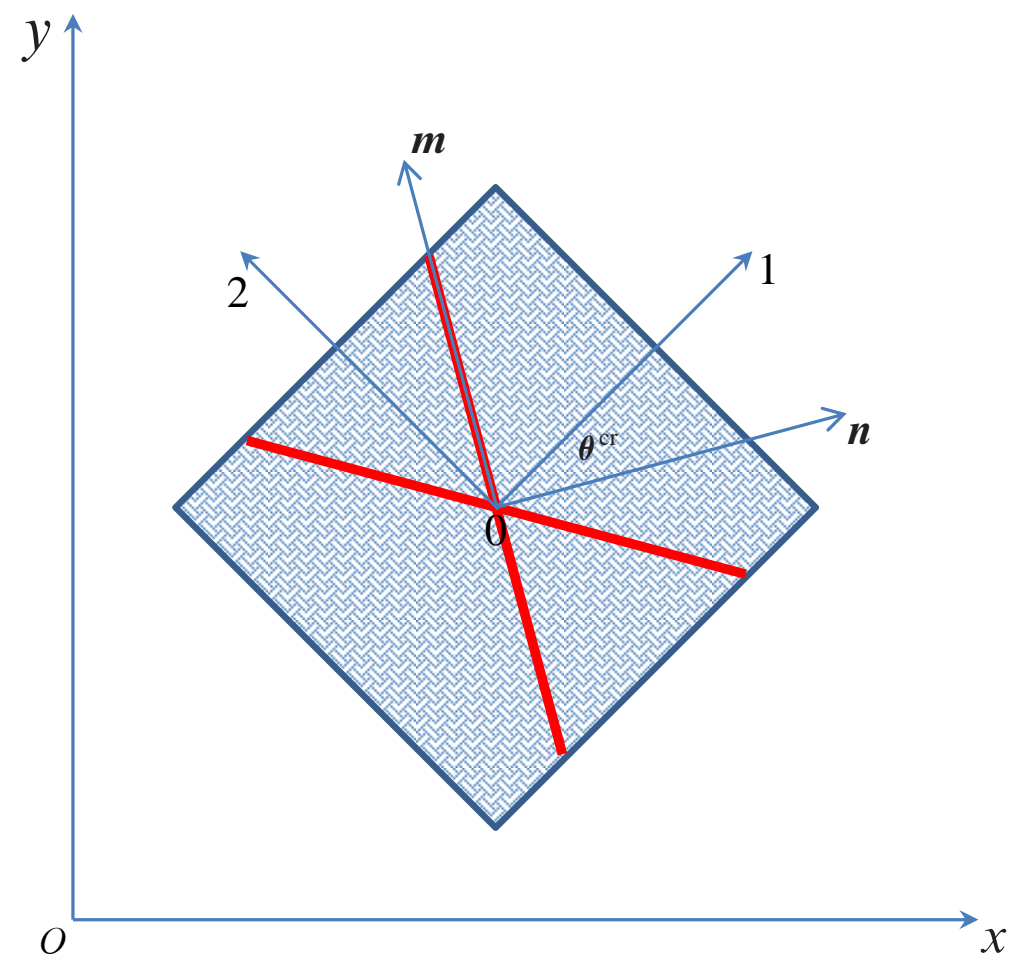

Fig. 3 Definition of the localization angle $\theta^{\text {cr }}$ between the normal vector $\boldsymbol{n}$ of the discontinuity and the material local axis 1 . 




Fig. 4 A strip under vertical stretching: Dimensions. The bottom and top edges are vertically stretched along opposite directions but with equal magnitude. 


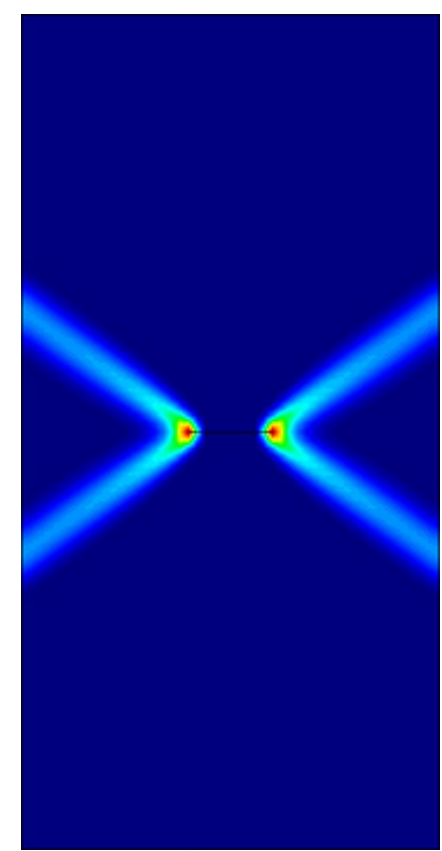

(a) $E_{11}=1.5 E_{0}$

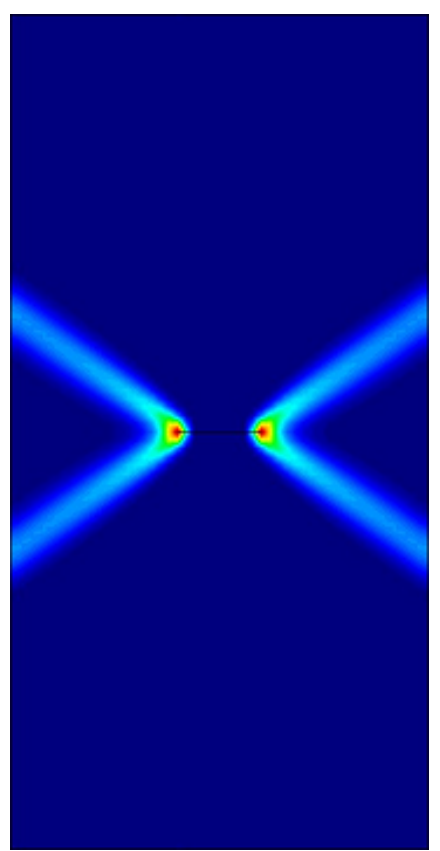

(b) $E_{11}=E_{22}=E_{0}$



(c) $E_{22}=1.5 E_{0}$

Fig. 5 A strip under vertical stretching (plane stress): Influence of Young's moduli on the localization angle $\theta^{\mathrm{cr}}=54.7^{\circ}$

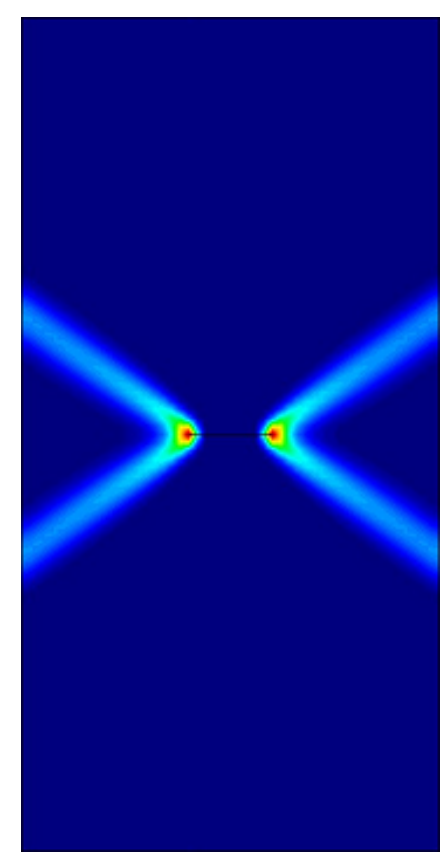

(a) $\nu_{0}=0.0$

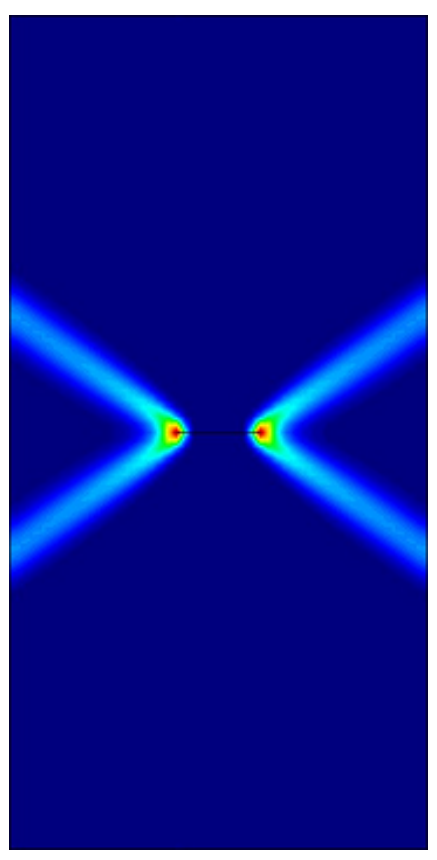

(b) $\nu_{0}=0.2$

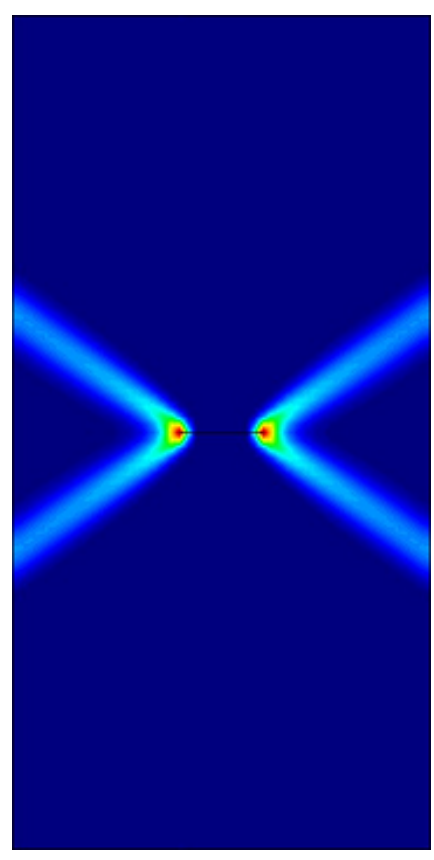

(c) $\nu_{0}=0.4$

Fig. 6 A strip under vertical stretching (plane stress): Influence of Poisson's ratio on the localization angle $\theta^{\text {cr }}=54.7^{\circ}$ 


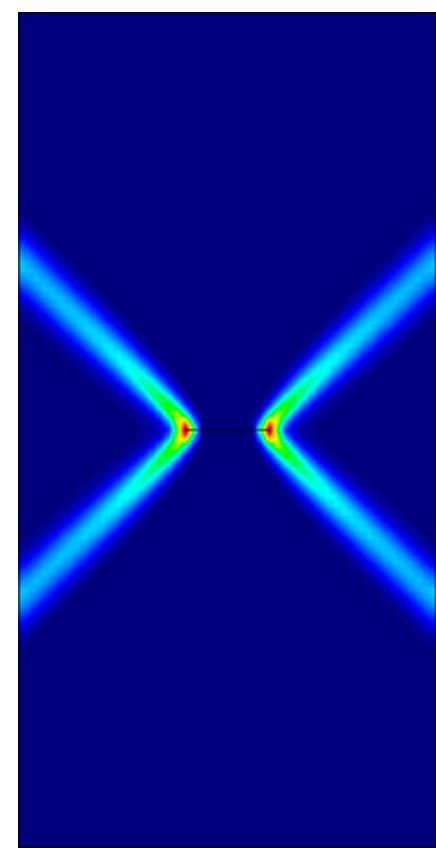

(a) $E_{11}=1.5 E_{0}$

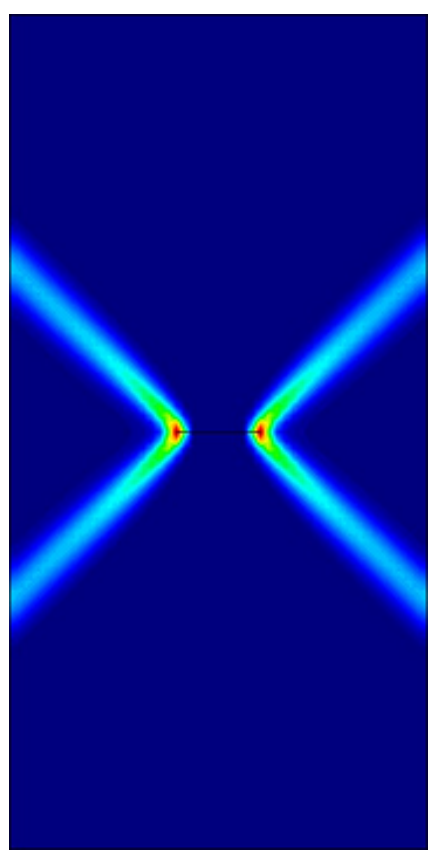

(b) $E_{11}=E_{22}=E_{0}$

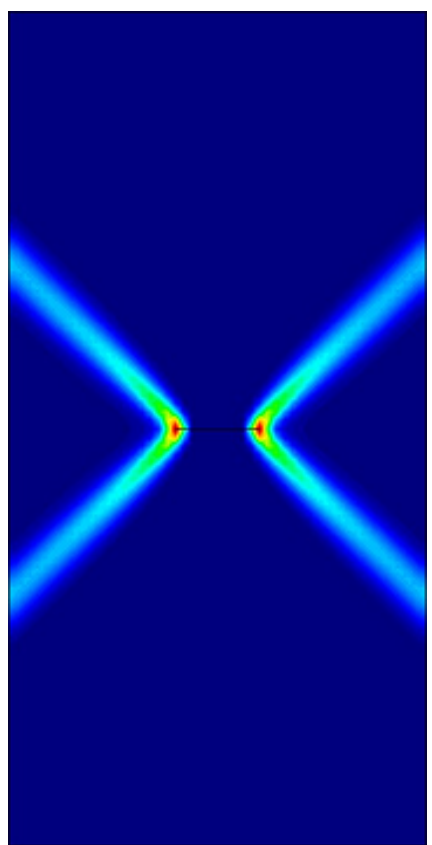

(c) $E_{22}=1.5 E_{0}$

Fig. 7 A strip under vertical stretching (plane strain): Influence of Young's moduli on the localization angle $\theta^{\text {cr }}=45^{\circ}$

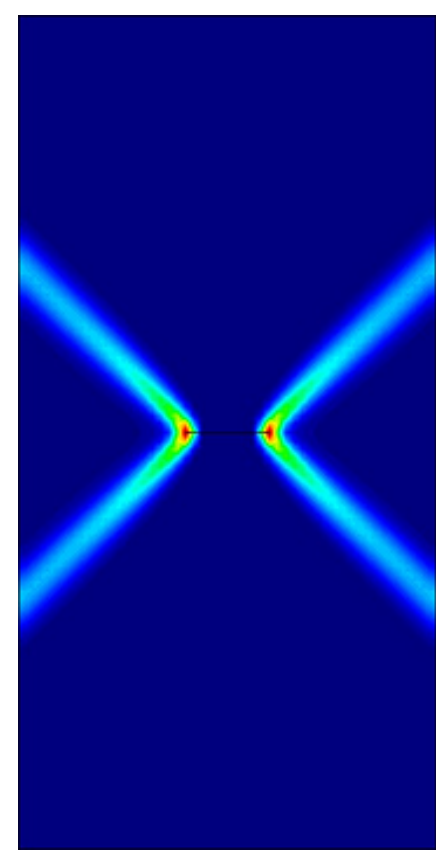

(a) $\nu_{0}=0.0$

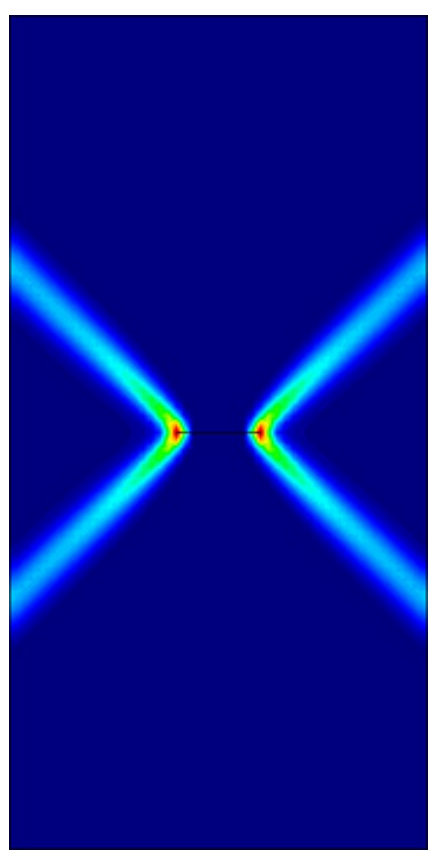

(b) $\nu_{0}=0.2$



(c) $\nu_{0}=0.4$

Fig. 8 A strip under vertical stretching (plane strain): Influence of Poisson's ratio on the localization angle $\theta^{\text {cr }}=45^{\circ}$ 




(a) $\sigma_{Y, 11}=0.75 \sigma_{Y}$

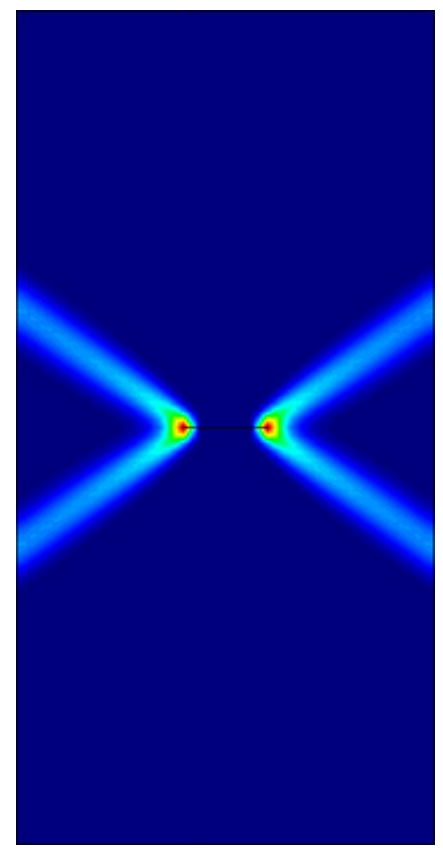

(d) $\sigma_{Y, 22}=0.75 \sigma_{Y}$

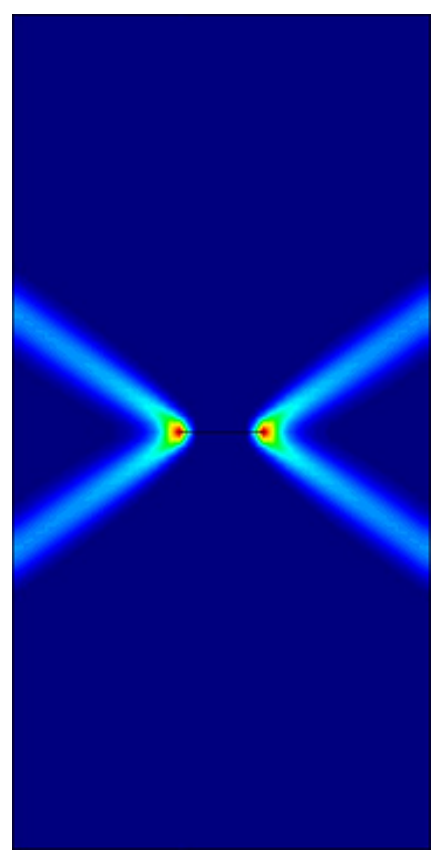

(b) $\sigma_{Y, 11}=\sigma_{Y}$



(e) $\sigma_{Y, 22}=\sigma_{Y}$

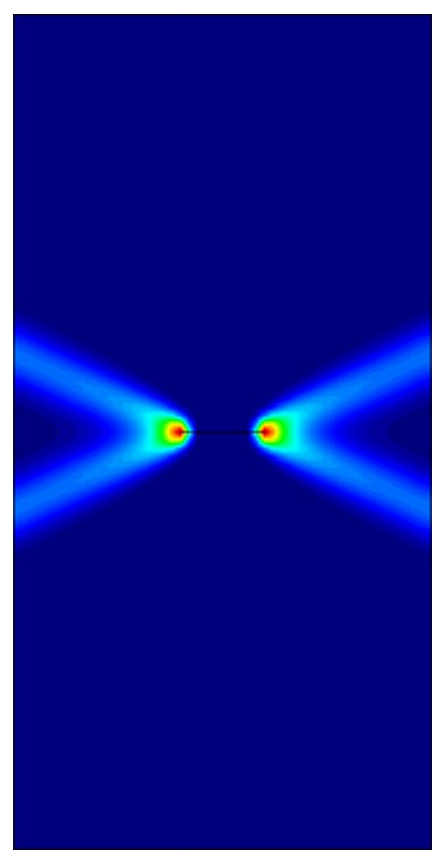

(c) $\sigma_{Y, 11}=1.50 \sigma_{Y}$

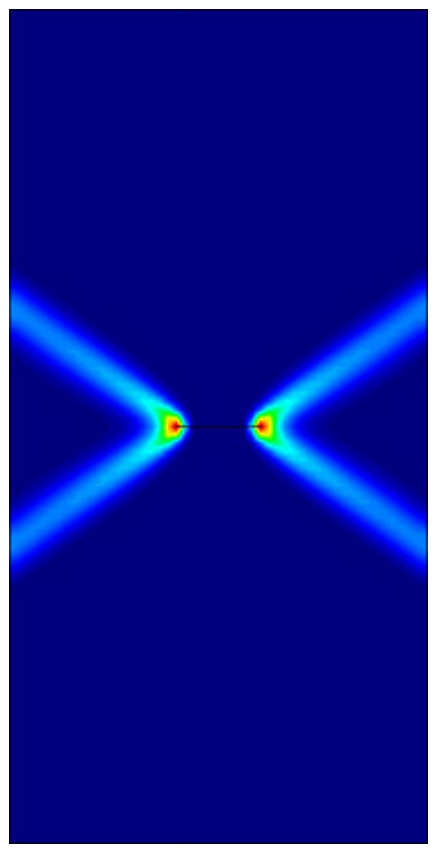

(f) $\sigma_{Y, 22}=1.50 \sigma_{Y}$

Fig. 9 A strip under vertical stretching (plane stress): Influence of the yield strengths $\sigma_{Y, 11}$ and $\sigma_{Y, 22}$ on the localization angle $\theta^{\mathrm{cr}}=54.7^{\circ}$ 


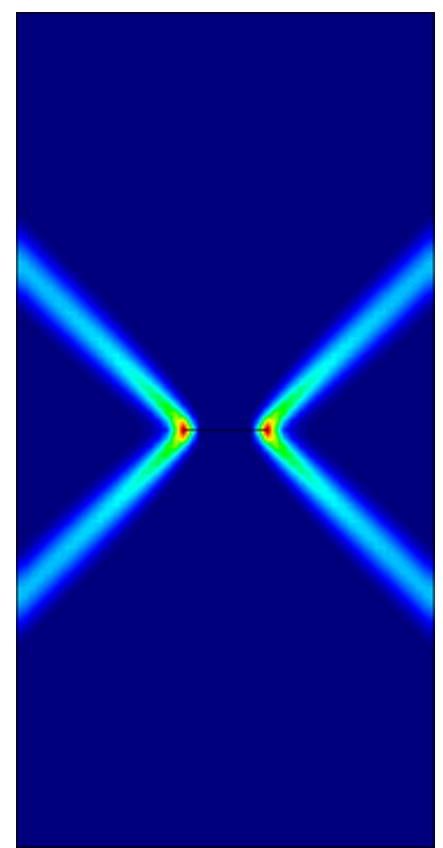

(a) $\sigma_{Y, 11}=0.75 \sigma_{Y}$

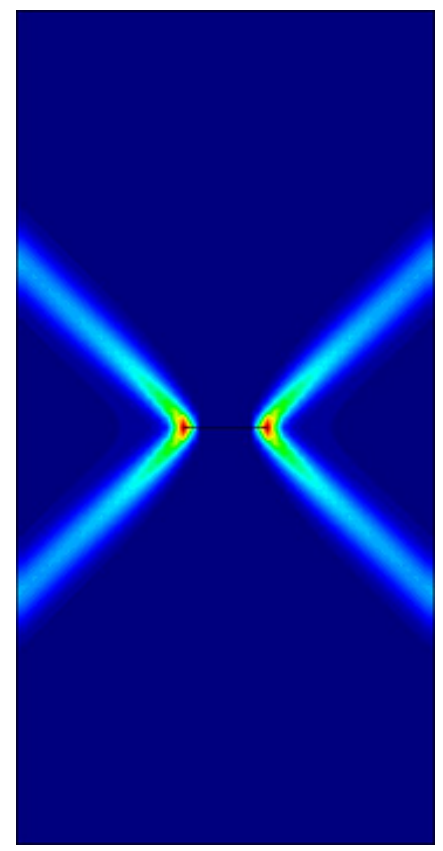

(d) $\sigma_{Y, 22}=0.75 \sigma_{Y}$



(b) $\sigma_{Y, 11}=\sigma_{Y}$

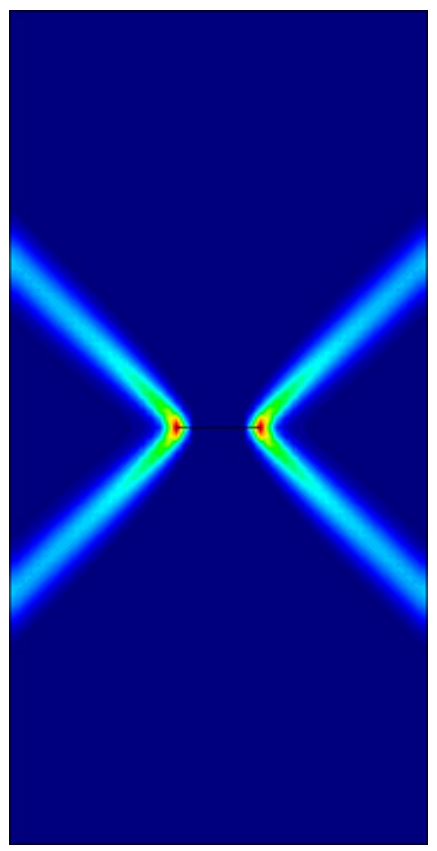

(e) $\sigma_{Y, 22}=\sigma_{Y}$

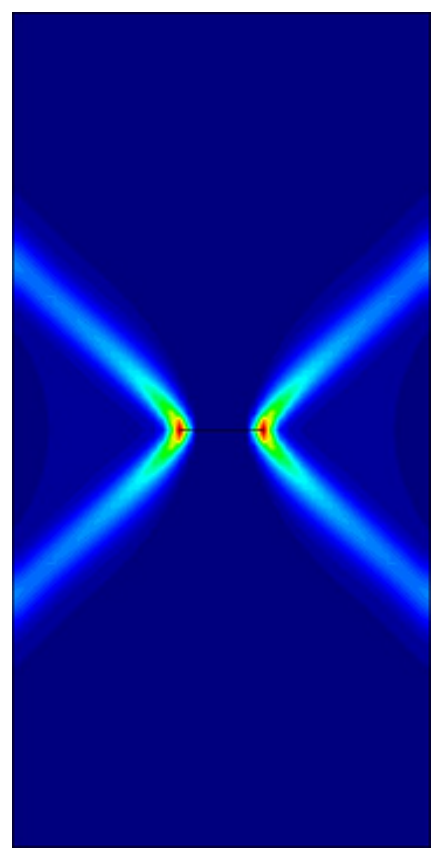

(c) $\sigma_{Y, 11}=1.50 \sigma_{Y}$

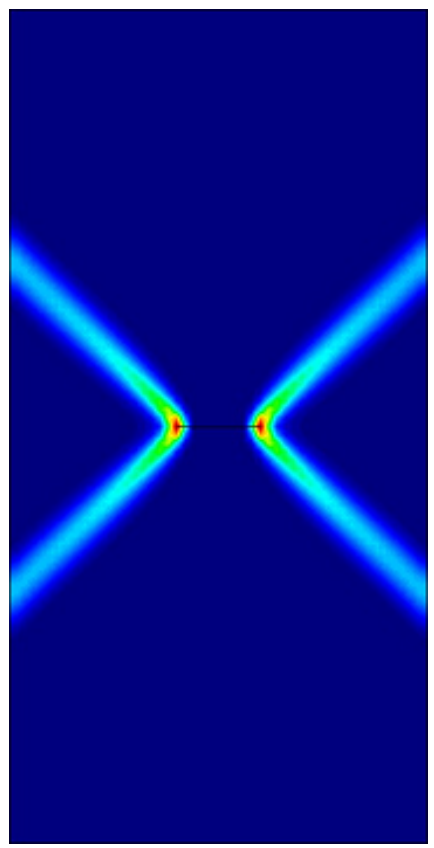

(f) $\sigma_{Y, 22}=1.50 \sigma_{Y}$

Fig. 10 A strip under vertical stretching (plane strain): Influence of the yield strength $\sigma_{Y, 11}$ and $\sigma_{Y, 22}$ on the localization angle $\theta^{\text {cr }}=45^{\circ}$. 




(a) $\alpha=0^{\circ}$

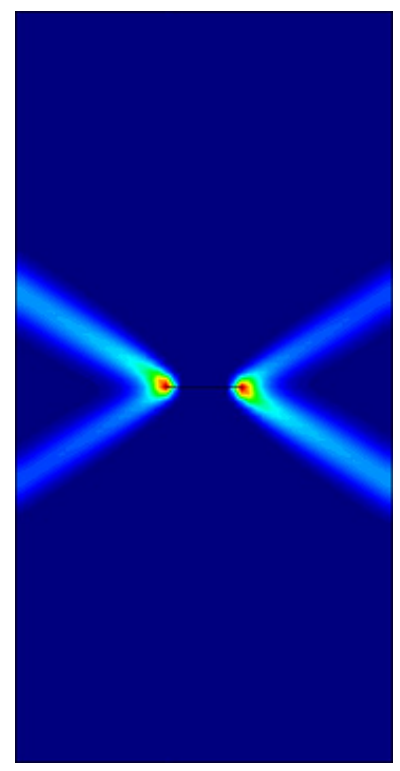

(b) $\alpha=30^{\circ}$

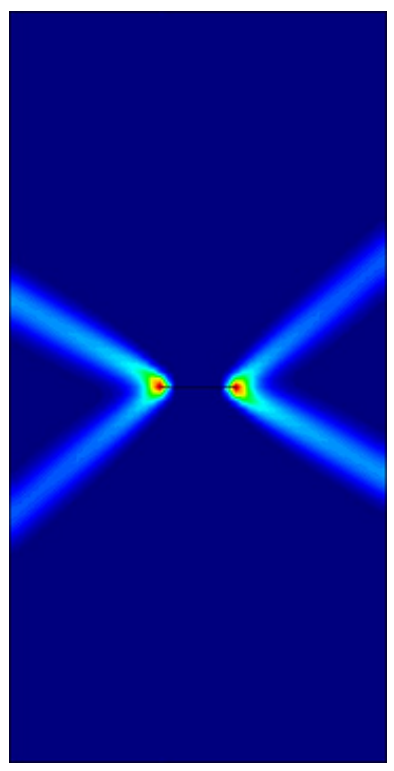

(c) $\alpha=45^{\circ}$

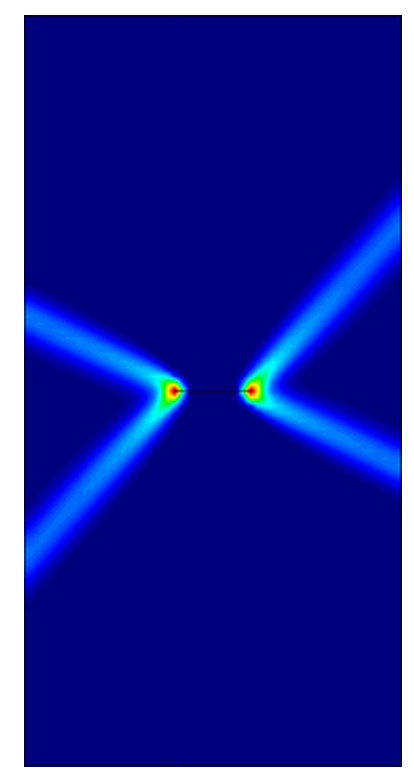

(d) $\alpha=60^{\circ}$

Fig. 11 A strip under vertical stretching (plane stress): Influence of the yield strength $\sigma_{Y, 11}=1.5 \sigma_{Y}$ on the localization angle $\theta^{\text {cr }}$.

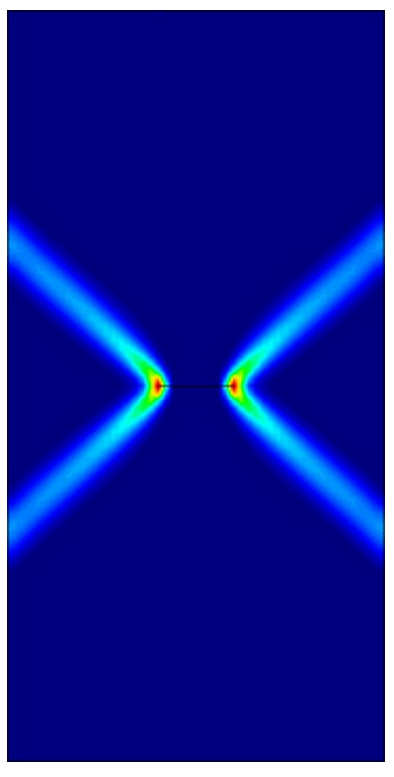

(a) $\alpha=0^{\circ}$

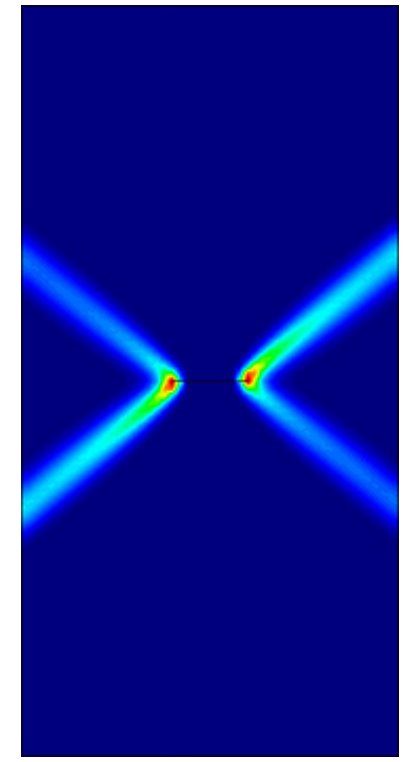

(b) $\alpha=30^{\circ}$

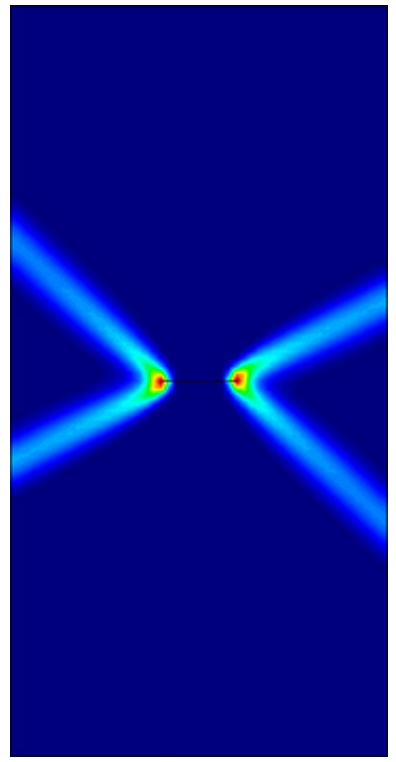

(c) $\alpha=45^{\circ}$

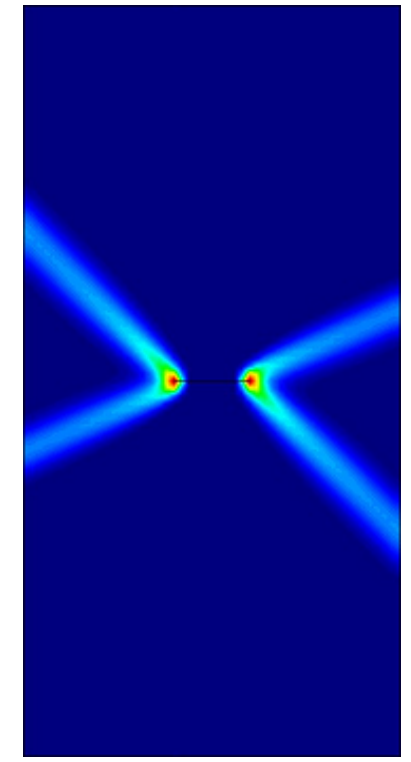

(d) $\alpha=60^{\circ}$

Fig. 12 A strip under vertical stretching (plane stress): Influence of the yield strength $\sigma_{Y, 11}=0.75 \sigma_{Y}$ on the localization angle $\theta^{\text {cr }}$ 


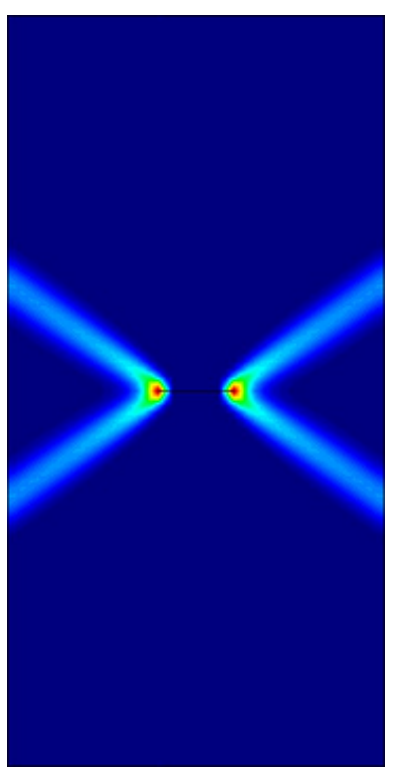

(a) $\alpha=0^{\circ}$

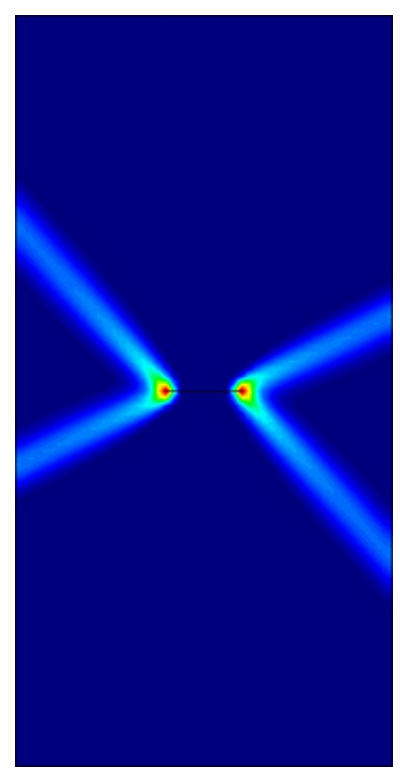

(b) $\alpha=30^{\circ}$

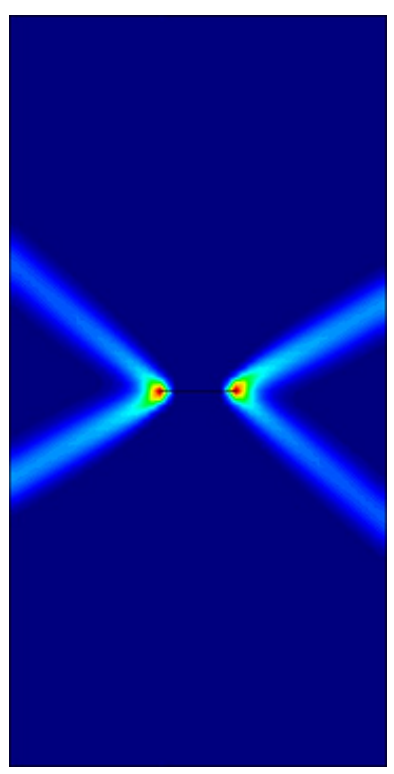

(c) $\alpha=45^{\circ}$

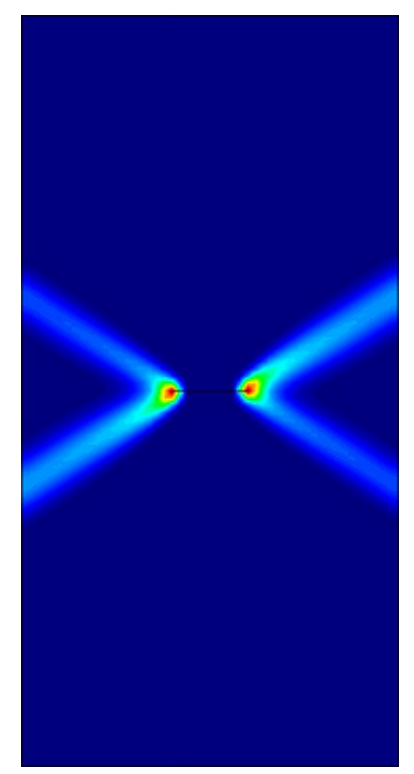

(d) $\alpha=60^{\circ}$

Fig. 13 A strip under vertical stretching (plane stress): Influence of the yield strength $\sigma_{Y, 22}=1.5 \sigma_{Y}$ on the localization angle $\theta^{\text {cr }}$.



(a) $\alpha=0^{\circ}$

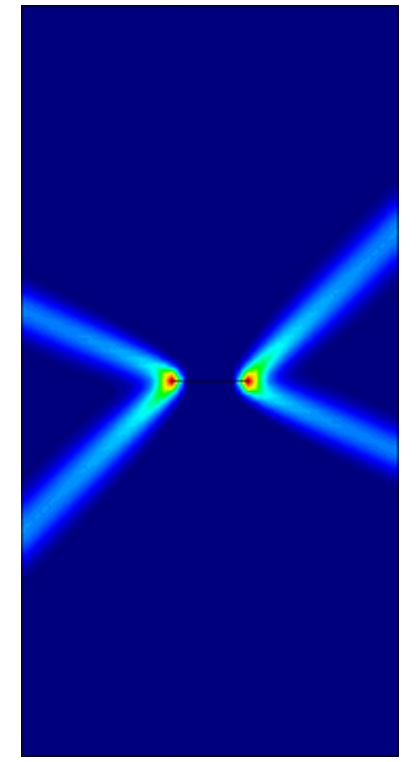

(b) $\alpha=30^{\circ}$

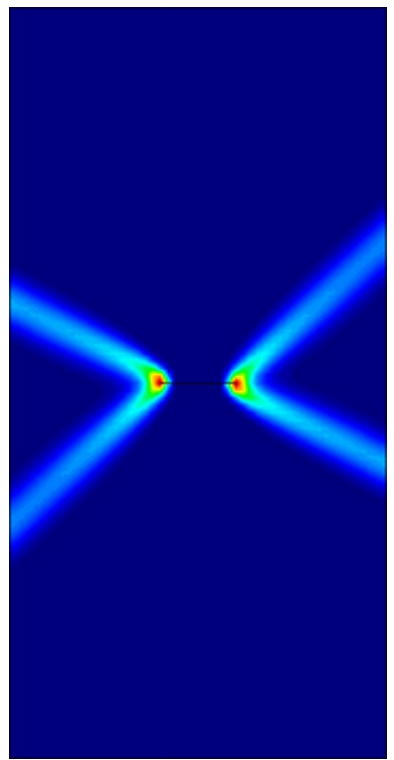

(c) $\alpha=45^{\circ}$

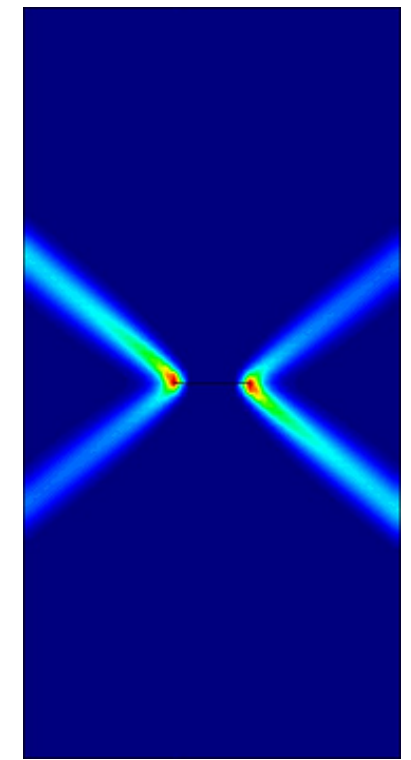

(d) $\alpha=60^{\circ}$

Fig. 14 A strip under vertical stretching (plane stress): Influence of the yield strength $\sigma_{Y, 22}=0.75 \sigma_{Y}$ on the localization angle $\theta^{\text {cr }}$ 


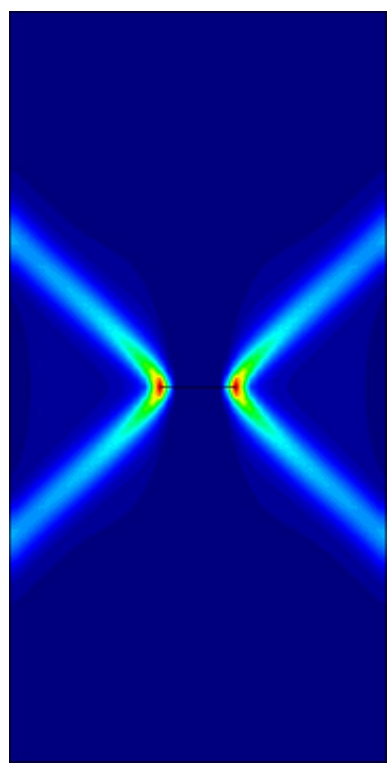

(a) $\alpha=0^{\circ}$

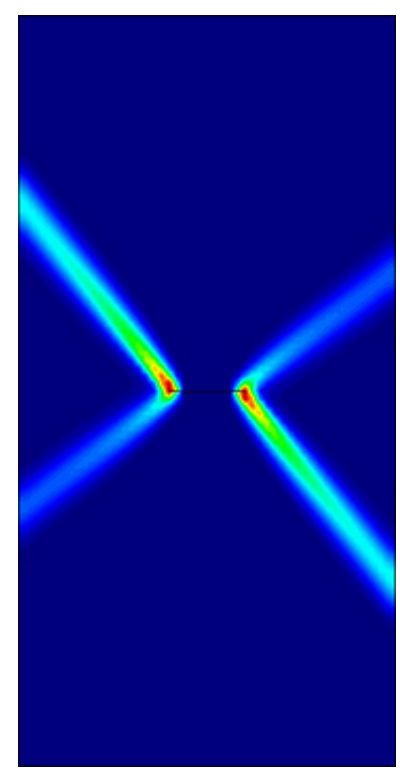

(b) $\alpha=30^{\circ}$

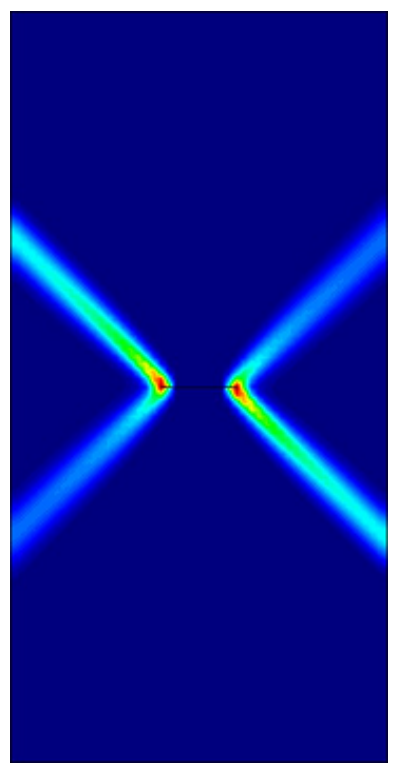

(c) $\alpha=45^{\circ}$



(d) $\alpha=60^{\circ}$

Fig. 15 A strip under vertical stretching (plane strain): Influence of the yield strength $\sigma_{Y, 11}=1.5 \sigma_{Y}$ or $\sigma_{Y, 22}=1.5 \sigma_{Y}$ on the localization angle $\theta^{\text {cr }}$.

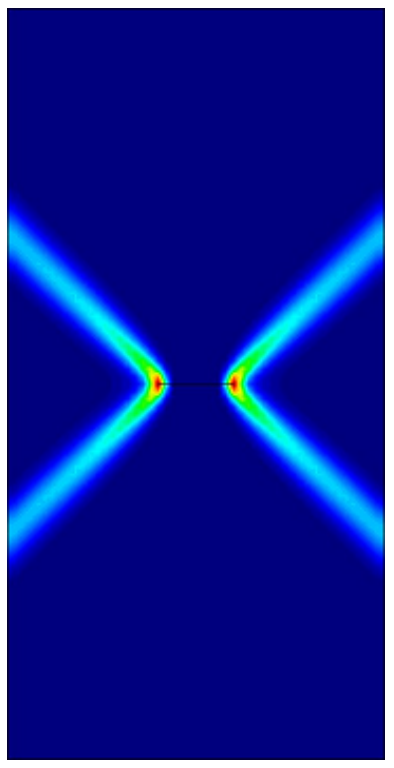

(a) $\alpha=0^{\circ}$

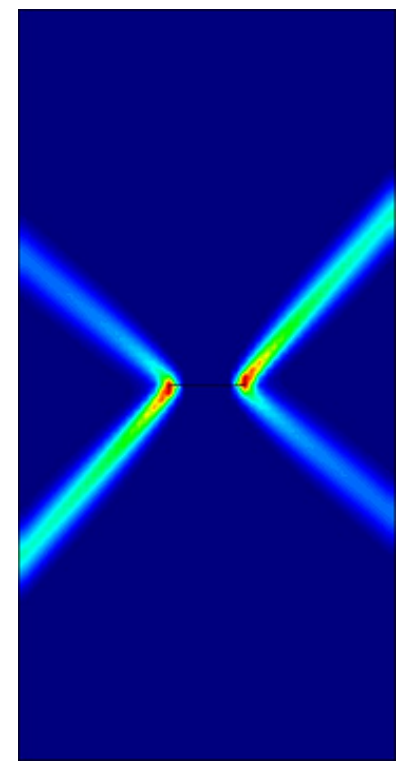

(b) $\alpha=30^{\circ}$

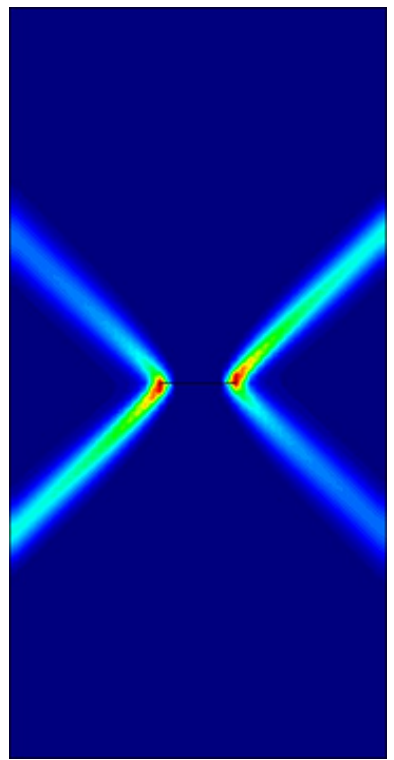

(c) $\alpha=45^{\circ}$

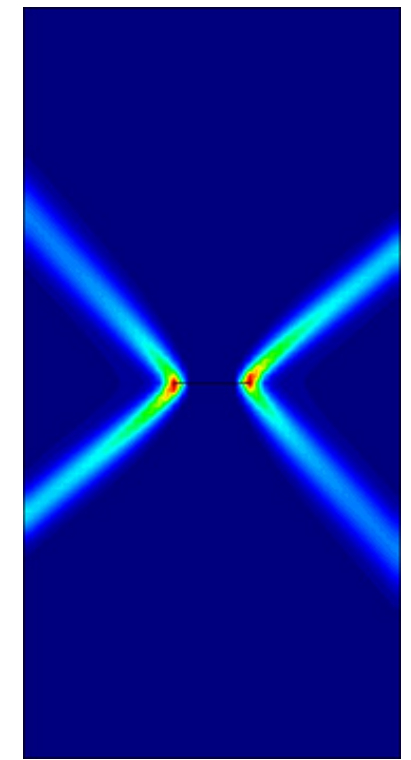

(d) $\alpha=60^{\circ}$

Fig. 16 A strip under vertical stretching (plane strain): Influence of the yield strength $\sigma_{Y, 11}=0.75 \sigma_{Y}$ or $\sigma_{Y, 22}=0.75 \sigma_{Y}$ on the localization angle $\theta^{\text {cr }}$. 


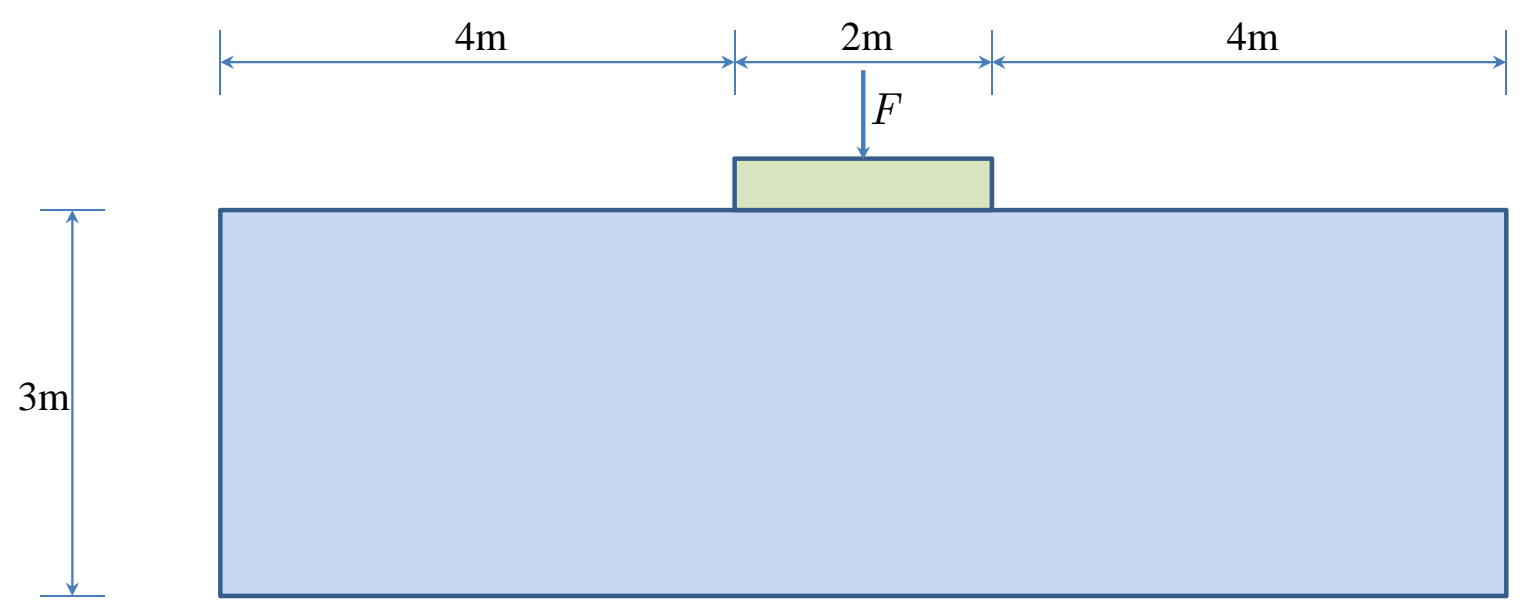

Fig. 17 Indentation by a flat rigid die: Dimensions and loading. The bottom edge is fixed in both direction, while the left and right edges are constrained along the horizontal direction.

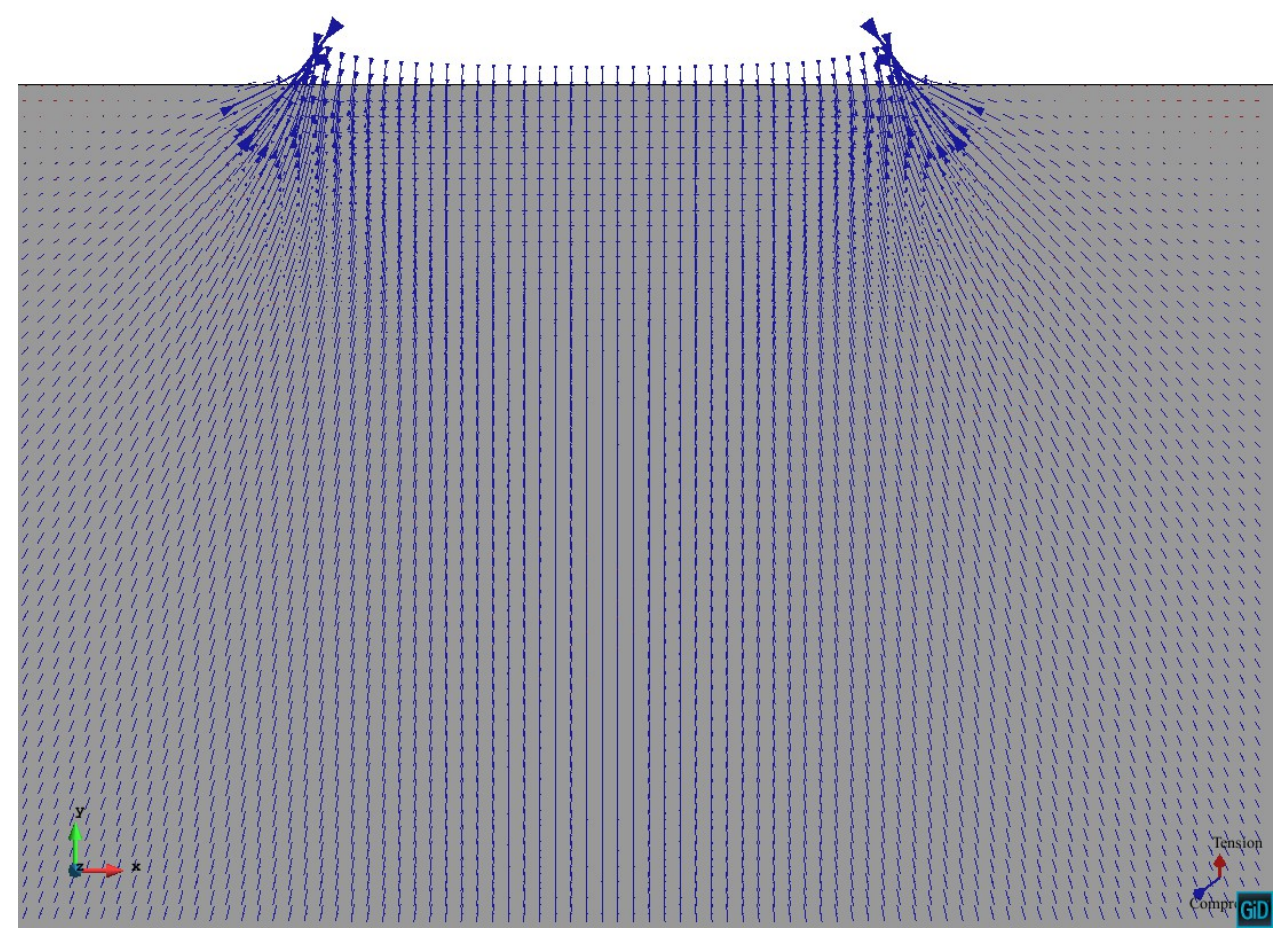

Fig. 18 Indentation by a flat rigid die: Directions of principal stresses around the rigid footing. 


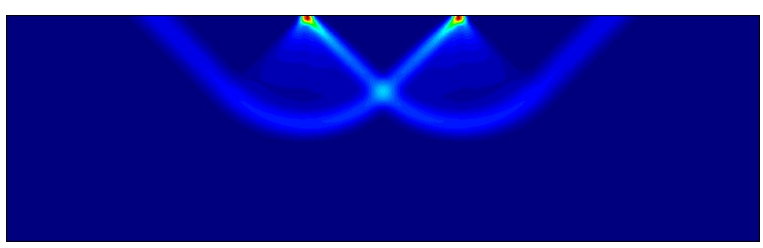

(a) $E_{11}=1.5 E_{0}$

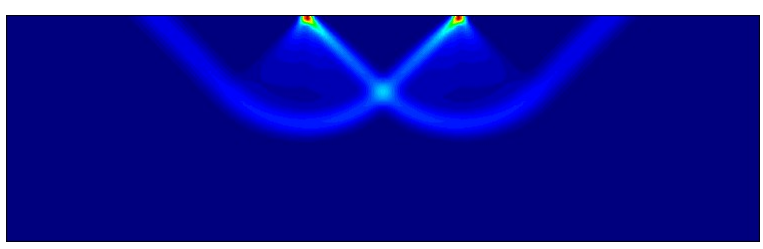

(c) $E_{11}=E_{22}=E_{0}$

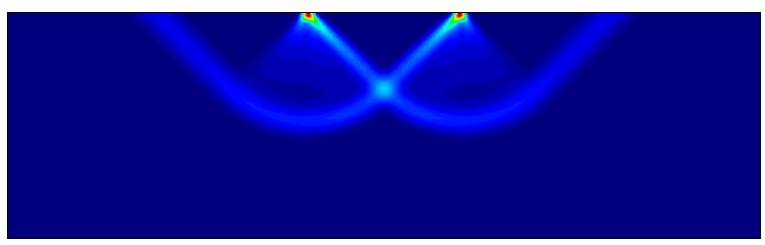

(e) $E_{22}=1.5 E_{0}$

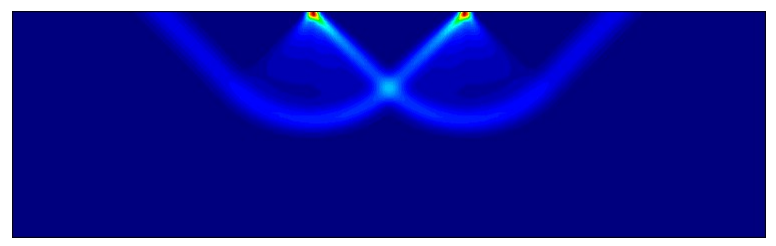

(b) $\nu_{0}=0.0$

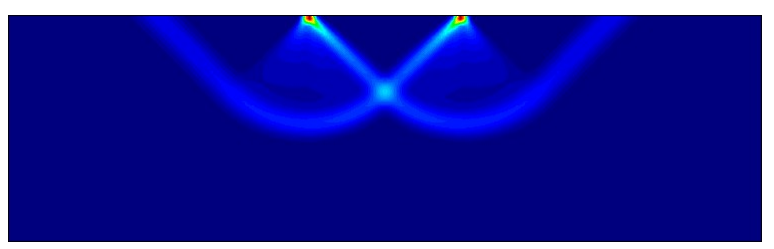

(d) $\nu_{0}=0.2$

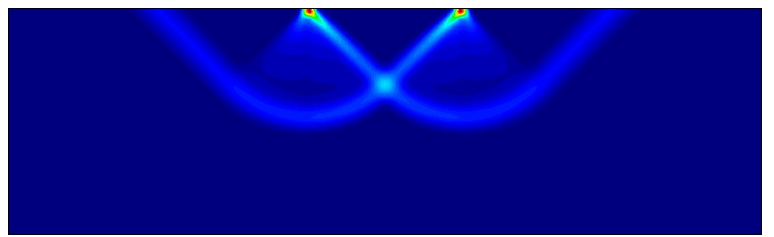

(f) $\nu_{0}=0.4$

Fig. 19 Indentation by a flat rigid die: Influence of Young's modulus and Poisson's ratio on the localization angle $\theta^{\text {cr }}$.



(a) $\sigma_{Y, 11}=0.75 \sigma_{Y}$

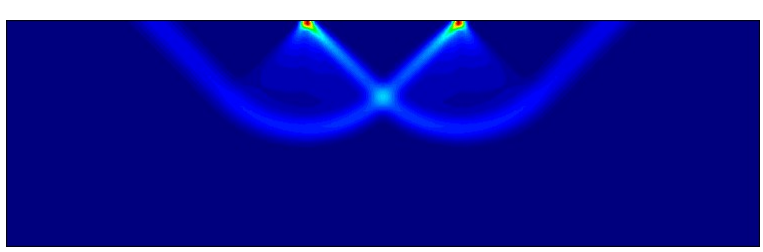

(c) $\sigma_{Y, 11}=\sigma_{Y}$

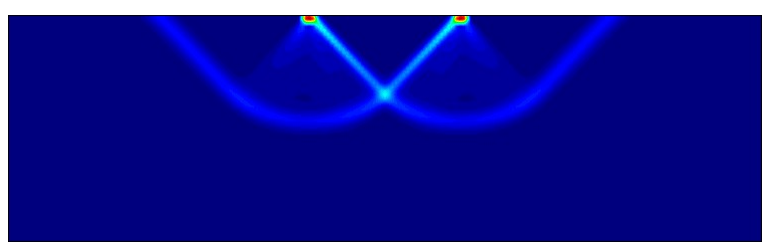

(e) $\sigma_{Y, 11}=1.50 \sigma_{Y}$

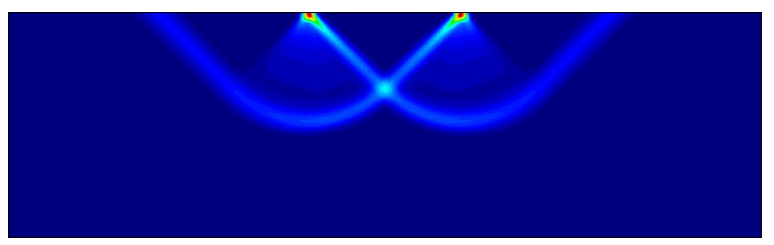

(b) $\sigma_{Y, 22}=0.75 \sigma_{Y}$

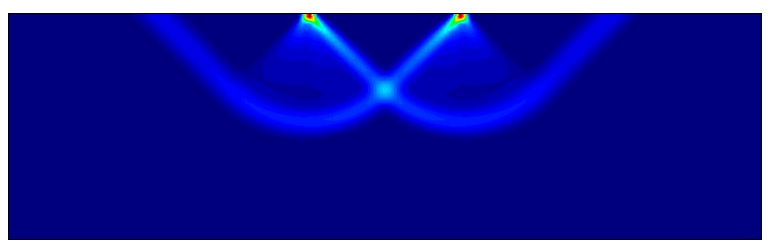

(d) $\sigma_{Y, 22}=\sigma_{Y}$



(f) $\sigma_{Y, 22}=1.50 \sigma_{Y}$

Fig. 20 Indentation by a flat rigid die: Influence of the material yield strengths $\sigma_{Y, 11}$ and $\sigma_{Y, 22}$ on the localization angle $\theta^{\text {cr }}$. 


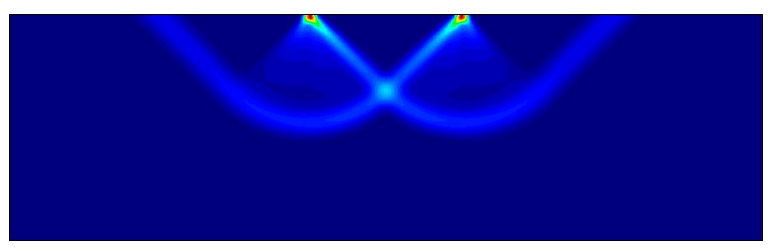

(a) $\alpha=0^{\circ}$

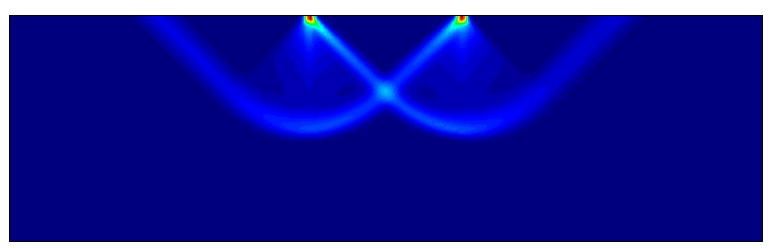

(c) $\alpha=45^{\circ}$

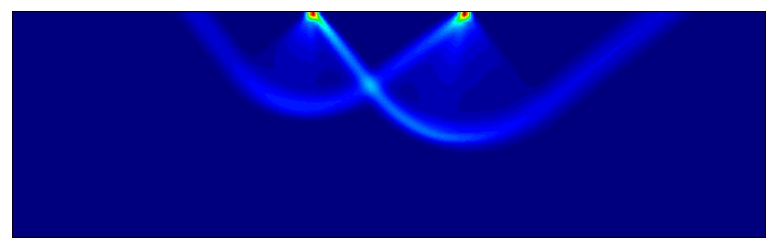

(b) $\alpha=30^{\circ}$

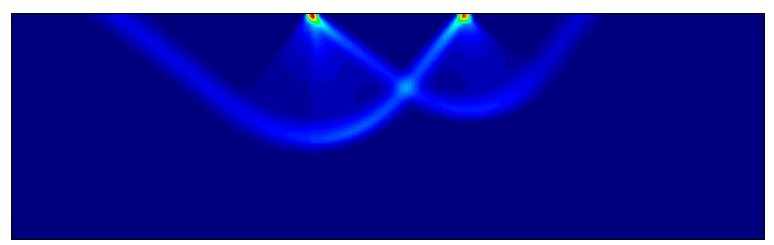

(d) $\alpha=60^{\circ}$

Fig. 21 Indentation by a flat rigid die: Influence of the material yield strengths $\sigma_{Y, 11}=1.5 \sigma_{Y}$ or $\sigma_{Y, 22}=1.5 \sigma_{Y}$ on the localization angle $\theta^{\text {cr }}$.

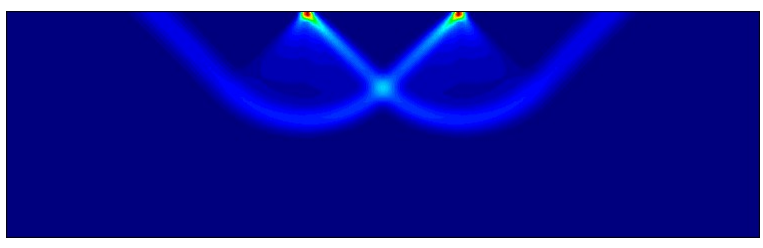

(a) $\alpha=0^{\circ}$

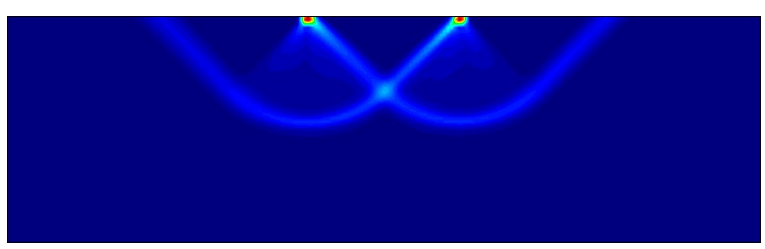

(c) $\alpha=45^{\circ}$

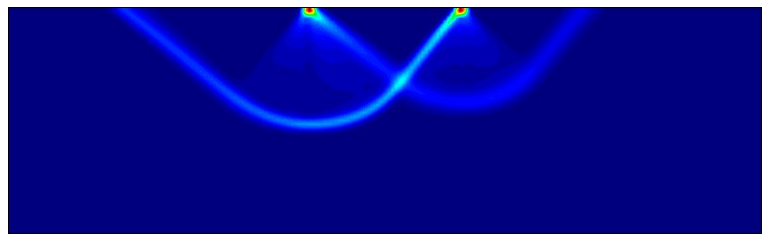

(b) $\alpha=30^{\circ}$

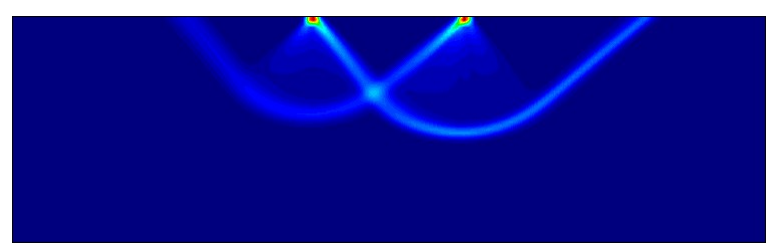

(d) $\alpha=60^{\circ}$

Fig. 22 Indentation by a flat rigid die: Influence of the material yield strengths $\sigma_{Y, 11}=0.75 \sigma_{Y}$ and $\sigma_{Y, 22}=0.75 \sigma_{Y}$ on the localization angle $\theta^{\text {cr }}$. 